JOURNAL OF

SYMPLECTIC GEOMETRY

Volume 6, Number 4, 353-378, 2008

\title{
REDUCTION OF BRANES IN GENERALIZED COMPLEX GEOMETRY
}

\author{
MARCO ZAMBON
}

We show that certain submanifolds of generalized complex manifolds ("weak branes") admit a natural quotient which inherits a generalized complex structure. This is analog to quotienting coisotropic submanifolds of symplectic manifolds. In particular, Gualtieri's generalized complex submanifolds ("branes") quotient to space-filling branes. Along the way, we perform reductions by foliations (i.e., no group action is involved) for exact Courant algebroids - interpreting the reduced Ševera class - and for Dirac structures.

\section{Contents}

1. Introduction 354

2. Review of Courant algebroids 355

3. The case of exact Courant algebroids 357

4. The case of Dirac structures 360

5. On the reduced Courant algebroid 362

5.1. Adapted splittings $\quad 362$

5.2. The Ševera class of the reduced Courant algebroid 364

6. The case of generalized complex structures 366

7. The case of (weak) branes 368

7.1. Reducing branes $\quad 368$

7.2. Reducing weak branes $\quad 372$

7.3. Cosymplectic submanifolds 375

$\begin{array}{ll}\text { References } & 376\end{array}$ 


\section{Introduction}

Consider the following setup in ordinary geometry: a manifold $M$ and a submanifold $C$ endowed with some integrable distribution $\mathcal{F}$ so that $\underline{C}:=$ $C / \mathcal{F}$ be smooth. Then we have a projection pr: $C \rightarrow \underline{C}$, which induces a vector bundle morphism $\mathrm{pr}_{*}: T C \rightarrow T \underline{C}$. If $M$ is endowed with some geometric structure, such as a symplectic 2 -form $\omega$, one can ask when $\omega$ induces a symplectic form on $\underline{C}$.

This happens for example when $C$ is a coisotropic submanifold ${ }^{1}$. Indeed, in this case, the pullback $i^{*} \omega$ of $\omega$ to $C$ has a kernel $\mathcal{F}$ which is of constant rank and integrable, and the closeness of $\omega$ ensures that if $p$ and $q$ lie in the same $\mathcal{F}$-leaf, then $\left(i^{*} \omega\right)_{p}$ and $\left(i^{*} \omega\right)_{q}$ project to the same linear symplectic form at $\operatorname{pr}(p)=\operatorname{pr}(q)$, so that one obtains a well-defined symplectic form on $\underline{C}$. An instance of the above is when there is a Lie group $G$ acting hamiltonianly on $M$ with moment map $\nu: M \rightarrow \mathfrak{g}^{*}$ and $C$ is the zero level set of $\nu$ (Marsden-Weinstein reduction [16]).

In this paper, we consider the geometry that arises when one replaces the tangent bundle $T M$ with an exact Courant algebroid $E$ over $M$ (any such $E$ is non-canonically isomorphic to $\left.T M \oplus T^{*} M\right)$. In this context, reduction by the action of a Lie group has been considered by several authors $[\mathbf{3}, \mathbf{1 0}, \mathbf{1 1}, \mathbf{1 4}, \mathbf{1 5}, \mathbf{1 8}]$; in this paper, we do not assume any group action. Unlike the tangent bundle case, knowing $\underline{C}$ does not automatically determine the exact Courant algebroid over it. We have to replace the foliation $\mathcal{F}$ by more data, namely a suitable subbundle $K$ of $\left.E\right|_{C}$, and we construct by a quotienting procedure a Courant algebroid $\underline{E}$ on $\underline{C}$ (Theorem 3.7). Our construction follows closely the one of $[\mathbf{3}]$, where the group action provides an identification between fibers of $E$ at different points; in our case, we make up for this by asking that there exist enough "basic sections" (Definition 3.3). Further, we describe in a simple way (see Definition 5.1) which splittings of $E$ induce 3-forms on $M$ (representing the Ševera class of $E$ ), which descend to 3 -forms on $\underline{C}$ (representing the Ševera class of $\underline{E}$ ).

Once we know how to reduce an exact Courant algebroid, we can ask when geometric structures defined on them descend to the reduced exact Courant algebroid. We consider Dirac structures (suitable subbundles of $E$ ) and generalized complex structures (suitable endomorphisms of $E$ ). We give sufficient conditions for these structures to descend in Proposition 4.1 and Proposition 6.1, respectively. The ideas and techniques are borrowed the literature cited above, in particular from $[\mathbf{3}, \mathbf{1 8}]$ (however our proof differs from these two references in that we reduce generalized complex structures directly and not viewing them as Dirac structures in the complexification of $E$ ).

\footnotetext{
${ }^{1}$ This means that the symplectic orthogonal of $T C$ is contained in $T C$.
} 
The heart of this paper is Section 7, where we identify the objects that automatically satisfy the assumptions needed to perform generalized complex reduction. When $M$ is a generalized complex manifold, we consider pairs consisting of a submanifold $C$ of $M$ and suitable maximal isotropic subbundle $L$ of $\left.E\right|_{C}$ (we call them "weak branes" in Definition 7.10). We show in Proposition 7.11 that weak branes admit a canonical quotient $\underline{C}$ which is endowed with an exact Courant algebroid and a generalized complex structure; this construction is inspired by Theorem 2.1 of Vaisman's work [20] in the setting of the standard Courant algebroid.

Particular cases of weak branes are generalized complex submanifolds $(C, L)$ (also known as "branes", see Definition 7.3), which were first introduced by Gualtieri $[\mathbf{7}]$ and are relevant to physics $[\mathbf{1 3}]$. Using our reduction of Dirac structures, we show in Theorem 7.4 that the quotients $\underline{C}$ of branes, which by the above are generalized complex manifolds, are also endowed with the structure of a space-filling brane. This is interesting also because space-filling branes induce an honest complex structure on the underlying manifold $[8]$.

The reduction statements we had to develop in order to prove the results of Section 7 are versions "without group action" of statements that already appeared in the literature $[\mathbf{2}, \mathbf{3}, \mathbf{1 0}, \mathbf{1 1}, \mathbf{1 4}, \mathbf{1 5}, \mathbf{1 8}, \mathbf{2 0}]$. Consequently, many ideas and techniques are borrowed from the existing literature; we make appropriate references in the text whenever possible. In particular, we followed closely [3] (also as far as notation and conventions are concerned).

Plan of the paper. In Section 2, we review exact Courant algebroids. In Section 3, we perform the reduction of exact Courant algebroids and determine objects that naturally satisfy the assumptions needed for the reduction. In Section 4, we perform the reduction of Dirac structures. In Section 5, we describe the reduced Ševera class. In Section 6, we reduce generalized complex structures and comment briefly on generalized Kähler reduction. The main section of this paper is Section 7: we reduce branes and weak branes, providing few examples. We also give a criterion that allows us to obtain weak branes by restricting to cosymplectic submanifolds.

\section{Review of Courant algebroids}

We review the notion of exact Courant algebroid; see $[\mathbf{3}, \mathbf{1 0}]$ for more details.

Definition 2.1. A Courant algebroid over a manifold $M$ is a vector bundle $E \rightarrow M$ equipped with a fiberwise non-degenerate symmetric bilinear form $\langle\cdot, \cdot\rangle$, a bilinear bracket $[\cdot, \cdot]$ on the smooth sections $\Gamma(E)$, and a bundle map $\pi: E \rightarrow T M$ called the anchor, which satisfy the following conditions for all $e_{1}, e_{2}, e_{3} \in \Gamma(E)$ and $f \in C^{\infty}(M)$ :

C1) $\left[e_{1},\left[e_{2}, e_{3}\right]\right]=\left[\left[e_{1}, e_{2}\right], e_{3}\right]+\left[e_{2},\left[e_{1}, e_{3}\right]\right]$, 
C2) $\pi\left(\left[e_{1}, e_{2}\right]\right)=\left[\pi\left(e_{1}\right), \pi\left(e_{2}\right)\right]$,

C3) $\left[e_{1}, f e_{2}\right]=f\left[e_{1}, e_{2}\right]+\left(\pi\left(e_{1}\right) f\right) e_{2}$,

C4) $\pi\left(e_{1}\right)\left\langle e_{2}, e_{3}\right\rangle=\left\langle\left[e_{1}, e_{2}\right], e_{3}\right\rangle+\left\langle e_{2},\left[e_{1}, e_{3}\right]\right\rangle$,

C5) $\left[e_{1}, e_{1}\right]=\mathcal{D}\left\langle e_{1}, e_{1}\right\rangle$,

where $\mathcal{D}=\frac{1}{2} \pi^{*} \circ d: C^{\infty}(M) \rightarrow \Gamma(E)$ (using $\langle\cdot, \cdot\rangle$ to identify $E$ with $E^{*}$ ).

We see from axiom C5 that the bracket is not skew-symmetric:

$$
\left[e_{1}, e_{2}\right]=-\left[e_{2}, e_{1}\right]+2 \mathcal{D}\left\langle e_{1}, e_{2}\right\rangle .
$$

Hence we have the following "Leibniz rule for the first entry": $\left[f e_{1}, e_{2}\right]=$ $f\left[e_{1}, e_{2}\right]-\left(\pi\left(e_{2}\right) f\right) e_{1}+2\left\langle e_{1}, e_{2}\right\rangle \mathcal{D} f$.

Definition 2.2. A Courant algebroid is exact if the following sequence is exact:

$$
0 \longrightarrow T^{*} M \stackrel{\pi^{*}}{\longrightarrow} E \stackrel{\pi}{\longrightarrow} T M \longrightarrow 0 .
$$

To simplify the notation, in the sequel we will often omit the map $T^{*} M \stackrel{\pi^{*}}{\rightarrow}$ $E^{*} \cong E$ and think of $T^{*} M$ as being a subbundle of $E$. Given an exact Courant algebroid, we may always choose a right splitting $\sigma: T M \rightarrow E$ whose image in $E$ is isotropic with respect to $\langle\cdot, \cdot\rangle$. Such a splitting induces the closed 3-form on $M$ given by

$$
H(X, Y, Z)=2\langle[\sigma X, \sigma Y], \sigma Z\rangle .
$$

Using the bundle isomorphism $\nabla+\frac{1}{2} \pi^{*}: T M \oplus T^{*} M \rightarrow E$, one can transport the Courant algebroid structure onto $T M \oplus T^{*} M$. The resulting structure is as follows (where $X_{i}+\xi_{i} \in \Gamma\left(T M \oplus T M^{*}\right)$ ): the bilinear pairing is

$$
\left\langle X_{1}+\xi_{1}, X_{2}+\xi_{2}\right\rangle=\frac{1}{2}\left(\xi_{2}\left(X_{1}\right)+\xi_{1}\left(X_{2}\right)\right),
$$

and the bracket is

$$
\left[X_{1}+\xi_{1}, X_{2}+\xi_{2}\right]_{H}=\left[X_{1}, X_{2}\right]+\mathcal{L}_{X_{1}} \xi_{2}-i_{X_{2}} d \xi_{1}+i_{X_{2}} i_{X_{1}} H
$$

which is the $H$-twisted Courant bracket on $T M \oplus T^{*} M[\mathbf{1 7}]$. Isotropic splittings of (2.1) differ by 2 -forms $b \in \Omega^{2}(M)$, and a change of splitting modifies the curvature $H$ by the exact form $d b$. Hence, there is a welldefined cohomology class $[H] \in H^{3}(M, \mathbb{R})$ attached to the exact Courant algebroid structure on $E$; $[H]$ is called the Ševera class of $E$.

We refer to $[\mathbf{3}, \mathbf{1 0}]$ for information on the group of automorphisms $\operatorname{Aut}(E)$ and its Lie algebra $\operatorname{Der}(E)$. Here we just mention few facts, the first of which underlies many of our constructions: for any $e \in \Gamma(E),[e, \cdot]$ is an element of $\operatorname{Der}(E)$ and hence integrates to an automorphism of the Courant algebroid $E$. Notice that for closed 1-forms $\xi$ (seen as sections of $T^{*} M \subset E$ ), we have $[\xi, \cdot]=0$ by $(2.3)$. Further, any 2 -form $B$ on $M$ determines a vector bundle 
$\operatorname{map} T M \oplus T M^{*} \rightarrow T M \oplus T M^{*}$ by $e^{B}: X+\xi \mapsto X+\xi+i_{X} B[\mathbf{7}]$ and these "gauge transformations" satisfy

$$
\left[e^{B} \cdot, e^{B} \cdot\right]_{H}=e^{B}[\cdot, \cdot]_{H+d B} .
$$

\section{The case of exact Courant algebroids}

In this section, we reduce exact Courant algebroids (Theorem 3.7).

Let $M$ be a manifold, $E$ an exact Courant algebroid over $M$, and $C$ a submanifold.

Lemma 3.1. Let $D \rightarrow C$ be a subbundle of $E$ such that $\pi\left(D^{\perp}\right) \subset T C$ (where $D^{\perp}$ denotes the orthogonal to $D$ w.r.t. the symmetric pairing), and $e_{1}, e_{2}$ sections of $D^{\perp}$. Then the expression $\left.\left[\tilde{e}_{1}, \tilde{e}_{2}\right]\right|_{C}$, where $\tilde{e}_{i}$ are extensions of $e_{i}$ to sections of $E \rightarrow M$, depends on the extensions only up to sections of $D$.

Proof. Fix extensions $\tilde{e}_{i}$ of $e_{i}(i=1,2)$. We have to show that for functions $f_{i}$ vanishing on $C$ and sections $\hat{e}_{i}$ of $E$, we have $\left.\left[\tilde{e}_{1}+f_{1} \hat{e}_{1}, \tilde{e}_{2}+f_{2} \hat{e}_{2}\right]\right|_{C}=$ $\left.\left[\tilde{e}_{1}, \tilde{e}_{2}\right]\right|_{C}$ up to sections of $D$. By the Leibniz rule C3 and since $\pi\left(e_{1}\right) \subset T C$, we have $\left.\left[\tilde{e}_{1}, f_{2} \hat{e}_{2}\right]\right|_{C}=0$. Also $\left.\left[f_{1} \hat{e}_{1}, \tilde{e}_{2}\right]\right|_{C}=\left.2\left\langle\hat{e}_{1}, \tilde{e}_{2}\right\rangle\left(\mathcal{D} f_{1}\right)\right|_{C} \subset N^{*} C \subset$ $\left(\pi\left(D^{\perp}\right)\right)^{\circ}=D \cap T^{*} M$. The term $\left.\left[f_{1} \hat{e}_{1}, f_{2} \hat{e}_{2}\right]\right|_{C}$ vanishes by the above since $\left.\left(f_{1} \hat{e}_{1}\right)\right|_{C}$ is a section of $D$.

Remark 3.2. If $D \rightarrow C$ is a subbundle of $E$ such that $\pi\left(D^{\perp}\right) \subset T C$, we can make sense of a statement like " $\left[e_{1}, e_{2}\right] \subset D$ " for $e_{1}, e_{2} \in \Gamma\left(D^{\perp}\right)$ : it means that $\left.\left[\tilde{e}_{1}, \tilde{e}_{2}\right]\right|_{C} \subset D$ for one (or equivalently, by Lemma 3.1, for all) extension $\tilde{e}_{i}$ to sections of $E \rightarrow M$. Similarly, we take $\left[\Gamma\left(D^{\perp}\right), \Gamma\left(D^{\perp}\right)\right] \subset \Gamma(D)$ to mean $\left[e_{1}, e_{2}\right] \subset D$ for all $e_{1}, e_{2} \in \Gamma\left(D^{\perp}\right)$.

Now fix an isotropic subbundle $K \rightarrow C$ of $E$, i.e., $K \subset K^{\perp}$, such that $\pi\left(K^{\perp}\right)=T C$.

Definition 3.3. We define the space of sections of $K^{\perp}$ which are basic w.r.t. $K$ as

$$
\Gamma_{\text {bas }}\left(K^{\perp}\right):=\left\{e \in \Gamma\left(K^{\perp}\right):[\Gamma(K), e] \subset \Gamma(K)\right\} .
$$

Remark 3.4. To ensure that a section $e$ of $K^{\perp}$ be basic, it suffices to consider locally defined sections of $K$ that span $K$ pointwise. That is, it suffices to show that for every point of $C$, there is a neighborhood $U \subset C$ and a subset $S \subset \Gamma\left(\left.K\right|_{U}\right)$ with $\operatorname{span}\left\{k_{p}: k \in S\right\}=K_{p}$ (for every $p \in U$ ) so that $\left[S,\left.e\right|_{U}\right] \subset \Gamma\left(\left.K\right|_{U}\right)$. Indeed, from the "Leibniz rule in the first entry", it follows that $[\Gamma(K), e] \subset \Gamma(K)$.

Lemma 3.5. Assume that the sections of $\Gamma_{\mathrm{bas}}\left(K^{\perp}\right)$ span $K^{\perp}$ at every point, i.e., that $\operatorname{span}\left\{e_{p}: e \in \Gamma_{\text {bas }}\left(K^{\perp}\right)\right\}=K_{p}^{\perp}$ for every $p \in C$. Then

1) $\left[\Gamma(K), \Gamma\left(K^{\perp}\right)\right] \subset \Gamma\left(K^{\perp}\right)$;

2) $[\Gamma(K), \Gamma(K)] \subset \Gamma(K)$. 
Proof. Fix a subset of sections $\left\{e_{i}\right\} \subset \Gamma_{\text {bas }}\left(K^{\perp}\right)$ that spans pointwise $K^{\perp}$. For any section $k$ of $K$ and functions $f_{i}$ (so that the sum $\sum f_{i} e_{i}$ is locally finite) by the Leibniz rule, we have $\left[k, \sum f_{i} e_{i}\right] \subset K^{\perp}$, proving 1 . Now 1 is equivalent to 2 , as can be seen using axiom $\mathrm{C} 4$ in the definition of Courant algebroid: let $k_{1}, k_{2}$ be sections of $K$ and $e$ a section of $K^{\perp}$. Then $\left\langle\left[k_{1}, e\right], k_{2}\right\rangle+\left\langle e,\left[k_{1}, k_{2}\right]\right\rangle=\pi\left(k_{1}\right)\left\langle e, k_{2}\right\rangle=0$ because $\pi(K) \subset \pi\left(K^{\perp}\right)=T C$.

Remark 3.6. A converse to Lemma 3.5 for local sections is given in [4].

The proof of the following theorem is modeled on Theorem 3.3 of [3]. When referring to the smoothness of the quotient of a manifold by a foliation, we refer to the unique differentiable structure so that the projection map is a submersion.

Theorem 3.7 (Exact Courant algebroid reduction). Let $E$ be an exact Courant algebroid over $M, C$ a submanifold of $M$, and $K$ an isotropic subbundle of $E$ over $C$ such that $\pi\left(K^{\perp}\right)=T C$. Assume that the space of (global) sections $\Gamma_{\text {bas }}\left(K^{\perp}\right)$ spans pointwise $K^{\perp}$ (i.e., that $\operatorname{span}\left\{e_{p}: e \in\right.$ $\left.\Gamma_{\text {bas }}\left(K^{\perp}\right)\right\}=K_{p}^{\perp}$ for every $p \in C$ ) and that the quotient $\underline{C}$ of $C$ by the foliation integrating $\pi(K)$ be a smooth manifold. Then there is an exact Courant algebroid $\underline{E}$ over $\underline{C}$ that fits in the following pullback diagram of vector bundles:

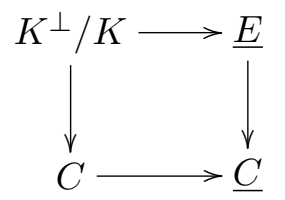

Proof. Notice that since $\pi(K)$ has constant rank iff $\pi\left(D^{\perp}\right)$ does (use, for example, equation $(2.17)$ of $[\mathbf{1 9}])$, it follows that $\pi(K)$ is a regular distribution on $C$. Further, by the assumption on basic sections and item 2 of Lemma $3.5, \pi(K)$ is an integrable distribution, so there exists a regular foliation integrating $\pi(K)$. We divide the proof into two steps.

Step 1. To describe the vector bundle $\underline{E}$, we have to explain how we identify fibers of $K^{\perp} / K$ over two points $p, q$ lying in the same leaf $F$ of $\pi(K)$. We do this as follows: we identify two elements $\hat{e}(p) \in\left(K^{\perp} / K\right)_{p}$ and $\hat{e}(q) \in\left(K^{\perp} / K\right)_{q}$ iff there is a section $e \in \Gamma_{\text {bas }}\left(K^{\perp}\right)$ which under the projection $K^{\perp} \rightarrow K^{\perp} / K$ maps $^{2}$ to $\hat{e}(p)$ at $p$ and $\hat{e}(q)$ at $q$. To show that this procedure gives a well-defined identification of $\left(K^{\perp} / K\right)_{p}$ and $\left(K^{\perp} / K\right)_{q}$, we need to show that if $e_{1}$ and $e_{2}$ are sections of $\Gamma_{\text {bas }}\left(K^{\perp}\right)$ such that $e_{1}(p)$

\footnotetext{
${ }^{2}$ In other words, we give a canonical trivialization of $\left.\left(K^{\perp} / K\right)\right|_{F}$ by projecting into it a frame for $\left.K^{\perp}\right|_{F}$ consisting of basic sections; by assumptions, we have enough basic sections to really get a frame for $\left.\left(K^{\perp} / K\right)\right|_{F}$.
} 
and $e_{2}(p)$ map to $\hat{e}(p)$, then $e_{1}(q)$ and $e_{2}(q)$ map to the same element of $\left(K^{\perp} / K\right)_{q}$.

Pick a finite sequence of local sections $k_{1}, \ldots, k_{n}$ of $K$ that join $p$ to $q$, i.e., such that following successively the vector fields $\pi\left(k_{i}\right)$ for times $t_{i}$, the point $p$ is mapped to $q$. Extend each $k_{i}$ to a section $\tilde{k}_{i}$ of $E$. Denote by $e^{a d_{\tilde{k}_{i}}}$ the Courant algebroid automorphism of $E$ obtained by integrating $a d_{\tilde{k}_{i}}=\left[\tilde{k}_{i}, \cdot\right]$, and by $\Phi$ the composition $e^{a d_{t_{n} \tilde{k}_{n}}} \circ \cdots \circ e^{a d_{t_{1} \tilde{k}_{1}}}$. Since $e_{1}$ is a basic section, we have $\left[k_{i}, e_{1}\right] \subset K$ for all $i$. So $\Phi\left(e_{1}(p)\right)-e_{1}(q) \in K_{q}$, and similarly for $e_{2}$. Now $e_{1}(p)-e_{2}(p) \in K_{p}$ by assumption, so because of item 2 of Lemma 3.5 we have $\Phi\left(e_{1}(p)-e_{2}(p)\right) \in K_{q}$. We deduce that $e_{1}(q)-e_{2}(q)$ also belong to $K_{q}$ and therefore project to the zero vector in $\left(K^{\perp} / K\right)_{q}$.

It is clear that $\underline{E}$, obtained from $K^{\perp} / K$ by identifying the fibers over each leaf of $\pi(K)$ as above, is endowed with a projection pr onto $\underline{C}$ (induced from the projection pr: $\left.K^{\perp} / K \rightarrow C\right)$. $\underline{E}$ is indeed a smooth vector bundle: given any point $\underline{p}$ of $\underline{C}$, choose a preimage $p \in C$ and a submanifold $S \subset C$ through $p$ transverse to the leaves of $\pi(K)$. $S$ provides a chart around $\underline{p}$ for the manifold $\underline{C}$, and $\operatorname{pr}^{-1}(S)$ is a vector subbundle of $K^{\perp} / K$ proving a chart for $\underline{E}$ around $p$.

Notice that pulling back by the vector bundle epimorphism $K^{\perp} / K \rightarrow \underline{E}$, we can embed the space of sections of $\underline{E}$ into the space of sections of $K^{\perp} / K$, the image being the image of $\Gamma_{\text {bas }}\left(K^{\perp}\right)$ under the map $K^{\perp} \rightarrow K^{\perp} / K$. In other words, we have a canonical identification $\Gamma(\underline{E}) \cong \Gamma_{\text {bas }}\left(K^{\perp}\right) / \Gamma(K)$.

Step 2. The pairing $\langle\cdot, \cdot\rangle$ on the fibers of $E$ induces a non-degenerate symmetric bilinear form on each fiber of $K^{\perp} / K$, which moreover descends to $\underline{E}$, because for any two given sections $e_{1}, e_{2} \in \Gamma_{\text {bas }}\left(K^{\perp}\right)$ and $k \in \Gamma(K)$, we have $\pi(k)\left\langle e_{1}, e_{2}\right\rangle=0$ using $\mathrm{C} 4$.

For the bracket of sections of $\underline{E}$, first notice that $\Gamma_{\text {bas }}\left(K^{\perp}\right)$ is closed (in the sense of Remark 3.2) under the bracket $[\cdot, \cdot]$ of $E$ : if $e_{1}, e_{2} \in \Gamma_{\text {bas }}\left(K^{\perp}\right)$, $\left[e_{1}, e_{2}\right]$ is a section of $K^{\perp}$ by the argument (using C4) in Theorem 3.3 of [3]. Further, $\left[e_{1}, e_{2}\right]$ is again basic by the "Jacobi identity" $\mathrm{C} 1$ : for any section $k$ of $K$, we have $\left[k,\left[e_{1}, e_{2}\right]\right]=\left[\left[k, e_{1}\right], e_{2}\right]+\left[e_{1},\left[k, e_{2}\right]\right]$. Now by definition of basic section, each $\left[k, e_{i}\right]$ lies in $K$, and applying once more the definition of basic section, ${ }^{3}$ we see that $\left[k,\left[e_{1}, e_{2}\right]\right] \subset K$, i.e., that $\left[e_{1}, e_{2}\right]$ is basic. In the light of Lemma 3.1, what we really have a well-defined bilinear form $\Gamma_{\text {bas }}\left(K^{\perp}\right) \times \Gamma_{\text {bas }}\left(K^{\perp}\right) \rightarrow \Gamma_{\text {bas }}\left(K^{\perp}\right) / \Gamma(K)$. Using the definition of basic section, we then have an induced bracket on $\Gamma_{\text {bas }}\left(K^{\perp}\right) / \Gamma(K)$, which as we saw is canonically isomorphic to $\Gamma(\underline{E})$.

The induced anchor map $\underline{\pi}: \underline{E} \rightarrow T \underline{C}$ is well-defined since $\pi(e)$ is a projectable vector field for any basic section $e \in \Gamma_{\text {bas }}\left(K^{\perp}\right)$, as follows using $\mathrm{C} 2$.

\footnotetext{
${ }^{3}$ Together with the fact that for any section $\hat{k}$ of $K$ we have $\left[e_{1}, \hat{k}\right]=-\left[\hat{k}, e_{1}\right]+2 \mathcal{D}\left\langle e_{1}, \hat{k}\right\rangle$ and $\mathcal{D}\left\langle e_{1}, \hat{k}\right\rangle \subset N^{*} C=K \cap T^{*} M$.
} 
It is straightforward to check that $\underline{E} \rightarrow \underline{C}$, endowed with the induced symmetric pairing, bilinear bracket on $\Gamma(\underline{E})$, and anchor $\underline{\pi}$, satisfies axioms C1-C5 in the definition of the Courant algebroid (Definition 2.1). Further, the proof of Theorem 3.3 of $[\mathbf{3}]$ shows that $\underline{E}$ is an exact Courant algebroid.

Remark 3.8. The subbundle $\left\{(e, \underline{e}) \mid e \in K^{\perp}\right\} \rightarrow\{(p, p) \mid p \in C\}$ of $E \times \underline{E} \rightarrow$ $M \times \underline{C}$ provides a morphism of Courant algebroids from $E$ to $\underline{E}$. Here we use (a slight variation of) the definition of morphism of Courant algebroids given in Definition 6.12 of [1] or in Definition 3.5.1 of [12].

Example 3.9 (Quotients of submanifolds). Take $E$ to be $T M \oplus T^{*} M$ with the untwisted bracket. Let $C$ be a submanifold endowed with a regular distribution $\mathcal{F}$, and assume that the quotient $\underline{C}=C / \mathcal{F}$ be smooth. Take $K:=\mathcal{F} \oplus N^{*} C . \quad \Gamma(K)$ is spanned by vector fields on $C$ lying in $\mathcal{F}$ and differentials of functions vanishing on $C$. Since the latter act trivially, it is enough to consider the action of a vector field $X \subset \mathcal{F}$. Let $\left.Y \oplus d f\right|_{C}$ be a section of $K^{\perp}$, where $Y$ is a projectable vector field and $f$ is the extension to $M$ of the pullback of a function on $\underline{C}$. The action of $X$ on this section is just $\left.[X, Y] \oplus\left(\mathcal{L}_{X} d f\right)\right|_{C}$, which lies again in $K$. Since such $\left.Y \oplus d f\right|_{C}$ span $K^{\perp}$, we can apply Theorem 3.7 and obtain a reduced Courant algebroid on $\underline{C}$, namely $T \underline{C} \oplus T^{*} \underline{C}$ with the untwisted bracket.

Example 3.10. Let $E$ be an exact Courant algebroid over $M$ and $C$ a submanifold of $M$. Then with $K=N^{*} C$, the assumptions of Theorem 3.7 are satisfied; indeed all the sections of $K^{\perp}=\pi^{-1}(T C)$ are basic. Hence, we recover Lemma 3.7 of $[\mathbf{3}]$.

\section{The case of Dirac structures}

Let $E$ be an exact Courant algebroid over $M$. Recall $[6]$ that a Dirac structure is a maximal isotropic subbundle of $E$ which is closed under the Courant bracket. Now we let $C$ be a submanifold of $M$ and consider a maximal isotropic subbundle $L \subset E$ defined over $C$ (not necessarily satisfying $\pi(L) \subset T C)$. The following is analog to Theorem 4.2 of $[\mathbf{3}]$.

Proposition 4.1 (Dirac reduction). Let $E \rightarrow M$ and $K \rightarrow C$ satisfy the assumptions of Theorem 3.7, so that we have an exact Courant algebroid $\underline{E} \rightarrow \underline{C}$. Let $L$ be a maximally isotropic subbundle of $\left.E\right|_{C}$ such that $L \cap K^{\perp}$ has constant rank, and assume that

$$
\left[\Gamma(K), \Gamma\left(L \cap K^{\perp}\right)\right] \subset \Gamma(L+K) .
$$

Then $L$ descends to a maximal isotropic subbundle $\underline{L}$ of $\underline{E} \rightarrow \underline{C}$. If furthermore

$$
\left[\Gamma_{\text {bas }}\left(L \cap K^{\perp}\right), \Gamma_{\text {bas }}\left(L \cap K^{\perp}\right)\right] \subset \Gamma(L+K),
$$


then $\underline{L}$ is an (integrable) Dirac structure. Here $\Gamma_{\text {bas }}\left(L \cap K^{\perp}\right):=\Gamma(L) \cap$ $\Gamma_{\text {bas }}\left(K^{\perp}\right)$.

Proof. At every $p \in C$, we have a Lagrangian relation between $E_{p}$ and $\left(K^{\perp} / K\right)_{p}$ given by $\left\{\left(e, e+K_{p}\right): e \in K_{p}^{\perp}\right\}$. The image of $L_{p}$ under this relation, which we denote by $\underline{L}(p)$, is maximal isotropic because $L_{p}$ is. Doing this at every point of $C$, we obtain a maximally isotropic subbundle of $K^{\perp} / K$, which is furthermore smooth because $L(p)$ is the image of ( $L \cap$ $\left.K^{\perp}\right)_{p}$, which has constant rank by assumption, under the projection $K_{p}^{\perp} \rightarrow$ $\left(K^{\perp} / K\right)_{p}$.

Recall that in Theorem 3.7, we identified $\left(K^{\perp} / K\right)_{p}$ and $\left(K^{\perp} / K\right)_{q}$ when $p$ and $q$ lie in the same leaf of $\pi(K)$. The identification is induced by the Courant algebroid automorphism $\Phi$ of $E$ obtained by integrating any sequence of locally defined sections $k_{1}, \ldots, k_{n}$ of $K$ that join $p$ to $q$. Assumption $(4.1)$ (with Lemma 3.5, 1) is exactly what is needed to ensure that $\Phi$ maps $L \cap K^{\perp}$ into $(L+K) \cap K^{\perp}=\left(L \cap K^{\perp}\right)+K$, so that $\underline{L}(p)$ gets identified with $\underline{L}(q)$. As a consequence, we obtain a well-defined smooth maximally isotropic subbundle $\underline{L}$ of the reduced Courant algebroid $\underline{E}$, i.e., an almost Dirac structure for $\underline{E}$. Now assume that (4.2) holds, and take two sections of $\underline{L}$, which by abuse of notation we denote $\underline{e}_{1}, \underline{e}_{2}$. Since $L \cap K^{\perp}$ has constant rank, we can lift them to sections $e_{1}, e_{2}$ of $\Gamma_{\text {bas }}\left(L \cap K^{\perp}\right)$. As for all elements of $\Gamma_{\text {bas }}\left(K^{\perp}\right)$, their bracket lies in $\Gamma_{\text {bas }}\left(K^{\perp}\right)$, and by assumption it also lies in $L+K$, so $\left[e_{1}, e_{2}\right]$ is a basic section of $(L+K) \cap K^{\perp}=\left(L \cap K^{\perp}\right)+K$. Its projection under $K^{\perp} / K \rightarrow \underline{E}$, which is by definition the bracket of $\underline{e}_{1}$ and $\underline{e}_{2}$, lies then in $\underline{L}$.

Example 4.2 (Coisotropic reduction). Let $(M, \Pi)$ be a Poisson manifold and $C$ a coisotropic submanifold ${ }^{4}$. It is known [6] that the characteristic distribution $\mathcal{F}:=\sharp N^{*} C$ is a singular integrable distribution; assume that it is regular and the quotient $\underline{C}=C / \mathcal{F}$ be smooth. It is known that $D=$ $\left\{(\sharp \xi, \xi): \xi \in T^{*} P\right\}$ is a Dirac structure for the untwisted Courant algebroid $T M \oplus T^{*} M$. By Example 3.9, choosing $K=\mathcal{F} \oplus N^{*} C$, we know that we can reduce this Courant algebroid and obtain the standard Courant algebroid on $\underline{C}$.

Using Proposition 4.1, now we show that $L:=\left.D\right|_{C}$ also descends. $L \cap K^{\perp}$ has constant rank since it's isomorphic to $\mathcal{F}^{\circ}$. To check (4.1), we use the fact that $K$ is spanned by closed 1-forms and hamiltonian vector fields of functions vanishing on $C$. The former act trivially, the latter (acting by Lie derivative) map $\Gamma(L)$ to itself because hamiltonian vector fields preserve the Poisson structure. An arbitrary section of $K$ maps $\left.\Gamma\left(L \cap K^{\perp}\right)\right]$ to $\Gamma(L+K)$ by the "Leibniz rule in the first entry" (see Section 2), so (4.1) is satisfied. Further it is known $[6]$ that the integrability of $\Pi$ is equivalent to $\Gamma(D)$ being

${ }^{4}$ This means that $\sharp N^{*} C \subset T C$, where $\sharp: T^{*} M \rightarrow T M$ is the contraction with $\Pi$. 
closed under the Courant bracket, so (4.2) holds. Hence, Proposition 4.1 tells us that $\Pi$ descends to a Dirac structure on $\underline{C}$. This of course is the Poisson structure obtained by the classical coisotropic reduction.

\section{On the reduced Courant algebroid}

Using the methods of Section 4, we derive some results on the reduced Courant algebroid obtained in Theorem 3.7. In this section, $E$ is an exact Courant algebroid over $M$ and $C$ a submanifold endowed with a coisotropic subbundle $K^{\perp}$ of $E$ satisfying $\pi\left(K^{\perp}\right)=T C$ and so that $\pi(K)$ is an integrable distribution with smooth quotient $\underline{C}:=C / \pi(K)$.

5.1. Adapted splittings. In this subsection, we consider "good" splittings of the exact Courant algebroid $E \rightarrow M$, and using their existence we determine simple data on a foliated submanifold that induce an exact Courant algebroid on the leaf space (Proposition 5.6).

Definition 5.1. We call a splitting $\sigma: T M \rightarrow E$ of the sequence (2.1) adapted to $K$ if

a) The image of $\sigma$ is isotropic;

b) $\sigma(T C) \subset K^{\perp}$;

c) for any vector field $X$ on $C$ which is projectable to $\underline{C}$, we have $\sigma(X) \in$ $\Gamma_{\text {bas }}\left(K^{\perp}\right)$.

Remark 5.2. For such a splitting, it follows automatically that $\sigma(\pi(K)) \subset$ $K$. Indeed by $\pi\left(K^{\perp}\right)=T C$, b in the definition above, and $K^{\perp} \cap T^{*} M=$ $(\pi(K))^{\circ}$, we have $K^{\perp}=\sigma(T C)+(\pi(K))^{\circ}$. Now $\langle\sigma(\pi(K)), \sigma(T C)\rangle=0$ by $\mathrm{a}$ in the definition above and $\left\langle\sigma(\pi(K)),(\pi(K))^{\circ}\right\rangle=0$. Hence $\sigma(\pi(K))$ has zero symmetric pairing with $K^{\perp}$.

Lemma 5.3. By the prescription $\sigma \mapsto L_{\sigma}:=\sigma(T M)$, splittings $\sigma$ adapted to $K$ correspond exactly to subbundles $L_{\sigma} \subset E$, with $\pi\left(L_{\sigma}\right)=T M$ satisfying

a) $L_{\sigma}$ is maximal isotropic;

b) $\pi\left(L_{\sigma} \cap K^{\perp}\right)=T C$;

c) $\left[\Gamma(K), \Gamma\left(L_{\sigma} \cap K^{\perp}\right)\right] \subset \Gamma\left(L_{\sigma}+K\right)$.

Proof. We just show that condition c is equivalent to item c in Definition 5.1. Assume condition c; we want to check that if $k \in \Gamma(K)$ and $X$ is a projectable vector field on $C$, then $[k, \sigma(X)] \in \Gamma(K)$. Since $\sigma(X) \subset L_{\sigma} \cap K^{\perp}$, this bracket is a section of $L_{\sigma}+K$. Further, since $\pi([k, \sigma(X)])=[\pi(k), X] \subset \pi(K)$ (because $X$ is projectable), it actually lies in $\left(L_{\sigma}+K\right) \cap \pi^{-1}(\pi(K))=K$.

The other implication follows because $L_{\sigma} \cap K^{\perp}=\sigma(T C)$ admits a frame of basic sections, namely $\sigma(X)$ as $X$ ranges over projectable vector fields on $C$. 
Lemma 5.4. Let $E$ be an exact Courant algebroid over a manifold $M, C$ a submanifold endowed with a regular foliation $\mathcal{F}$ so that $C / \mathcal{F}$ is smooth, and $L$ a maximal isotropic subbundle $\left.L \subset E\right|_{C}$ with $\pi(L)=T C$ such that $[\Gamma(K), \Gamma(L)] \subset \Gamma(L)$ where $K:=L \cap \pi^{-1}(\mathcal{F})$. Then there exists a splitting adapted to $K$.

Proof. Notice that $K$ is isotropic and has constant rank, because $\operatorname{ker}\left(\left.\pi\right|_{K}\right)=$ $K \cap T^{*} M=L \cap T^{*} M=N^{*} C$ has constant rank and $\pi(K)=\mathcal{F}$ has constant rank by assumption. Also $K^{\perp}=L+\mathcal{F}^{\circ}$, so $\pi\left(K^{\perp}\right)=T C$. Let $\sigma: T M \rightarrow E$ be an isotropic splitting such that $\sigma(T C) \subset L$. Then $L_{\sigma}:=\sigma(T M)$ clearly satisfies conditions a and $\mathrm{b}$ in Lemma 5.3. Further, it satisfies condition c because $L_{\sigma} \cap K^{\perp}+K=L$. Hence, by Lemma 5.3, $\sigma$ is a splitting adapted to $K$.

The following proposition says that splittings adapted to $K$ exist if and only if the reduced exact Courant algebroid $\underline{E}$ as in Theorem 3.7 exists.

Proposition 5.5. Let $K \rightarrow C$ be an isotropic subbundle of $E$ with $\pi\left(K^{\perp}\right)=$ $T C$ and assume that $\pi(K)$ be integrable and $\underline{C}:=C / \pi(K)$ be smooth. Then splittings adapted to $K$ exist if and only if $\Gamma_{\text {bas }}\left(K^{\perp}\right)$ spans $K^{\perp}$ at every point of $C$.

Proof. Assume first that a splitting $\sigma$ adapted to $K$ exists. Let $X$ be a projectable vector field on $C$. By c of Definition 5.1, $\sigma(X)$ will lie in $\Gamma_{\text {bas }}\left(K^{\perp}\right)$. Take a function on $\underline{C}$, pull it back to a function $C$, and extend it to a function $f$ on $M$. Then $\left.d f\right|_{C}$ is a section of $(\pi(K))^{\circ}=T^{*} M \cap K^{\perp}$. Further, it lies in $\Gamma_{\text {bas }}\left(K^{\perp}\right)$ : for any $k \in \Gamma(K)$, we have

$$
\left[k,\left.d f\right|_{C}\right]=-\left[\left.d f\right|_{C}, k\right]+d\left\langle k,\left.d f\right|_{C}\right\rangle \subset N^{*} C \subset K
$$

because $d f$ as a closed 1-form acts trivially and it annihilates $\pi(K)$. Since $K^{\perp}=\sigma(T C)+\left(T^{*} M \cap K^{\perp}\right)$, taking all projectable vector fields $X$ and functions $f$ as above, we see that $\Gamma_{\text {bas }}\left(K^{\perp}\right)$ spans $K^{\perp}$ at every point of $M$.

Conversely, assume now that $\Gamma_{\text {bas }}\left(K^{\perp}\right)$ spans $K^{\perp}$ at every point of $C$. Then by Theorem 3.7, the reduced Courant algebroid $\underline{E}$ over $\underline{C}$ exists; let $\underline{\sigma}: T \underline{C} \rightarrow \underline{E}$ be any isotropic splitting. Denote by $L$ the preimage of the maximal isotropic subbundle $\underline{\sigma}(T \underline{C})$ under $p: K^{\perp} \rightarrow K^{\perp} / K \rightarrow \underline{E}$. $L$ is a maximal isotropic subbundle of $K^{\perp}$, and $\pi(L)=T C$. Furthermore, lifting sections of $\underline{\sigma}(T \underline{C})$ to basic sections of $K^{\perp}$, we see that $\Gamma_{\text {bas }}(L)$ (the basic sections that lie in $L$ ) spans $L$ at every point of $C$, hence from $\left[\Gamma(K), \Gamma_{\text {bas }}(L)\right] \subset \Gamma(K)$ we can conclude $[\Gamma(K), \Gamma(L)] \subset \Gamma(L)$. Notice also that $L \cap \pi^{-1}(\pi(K))=p^{-1}\left(\underline{\sigma}(T \underline{C}) \cap T^{*} \underline{C}\right)=K$. Hence we can apply Lemma 5.4 and obtain a splitting of $E$ adapted to $K$. 
Putting together Lemma 5.4, Proposition 5.5, and Theorem 3.7, we are obtain:

Proposition 5.6. Let $E$ be an exact Courant algebroid over a manifold $M, C$ a submanifold endowed with a regular integrable foliation $\mathcal{F}$ so that $C / \mathcal{F}$ be smooth, and $L$ a maximal isotropic subbundle $\left.L \subset E\right|_{C}$ with $\pi(L)=$ $T C$ such that $[\Gamma(K), \Gamma(L)] \subset \Gamma(L)$ where $K:=L \cap \pi^{-1}(\mathcal{F})$. Then $E$ descends to an exact Courant algebroid on $C / \mathcal{F}$.

5.2. The Ševera class of the reduced Courant algebroid. In Theorem 3.7 , we showed that, when certain assumptions are met, one obtains an exact Courant algebroid $\underline{E}$ over the quotient $\underline{C}$ of $C$ by the distribution $\pi(K)$. In this subsection, we will discuss how to obtain the Ševera class of $\underline{E}$ from the one of $E$.

We start observing that if $\sigma$ is a splitting adapted to $K$, then $j^{*} H_{\sigma}$ descends to a 3 -form on $\underline{C}$, where $j$ is the inclusion of $C$ in $M$. Since $H_{\sigma}$ is closed, we just need to check $i_{X}\left(j^{*} H_{\sigma}\right)=0$, where $X \in \pi(K)$. Extend $X$ to a vector field tangent to $\pi(K)$; take vectors $Y, Z \in T_{p} C$ and extend them locally to projectable vector fields of $C$. Since $\sigma$ is an splitting adapted to $K$, we know that $\sigma(Y) \in \Gamma_{\text {bas }}\left(K^{\perp}\right)$, and since $\sigma(X) \subset K$ (by Remark $5.2)$, we have $[\sigma(X), \sigma(Y)] \subset K$. Therefore, indeed,

$$
H_{\sigma}(X, Y, Z)=2\langle[\sigma(X), \sigma(Y)], \sigma(Z)\rangle=0 .
$$

Even more is true by the following, which is an analog of Proposition 3.6 of [3] (but unlike that proposition does not involve equivariant cohomology; see also $[15,14])$.

Proposition 5.7. If $\sigma$ is a splitting adapted to $K$, then $j^{*}\left(H_{\sigma}\right)$ descends to a closed 3-form on $\underline{C}$, which represents the Ševera class of $\underline{E}$.

Proof. Since $\sigma$ is an adapted splitting, $L_{\sigma}:=\sigma(T M)$ satisfies the conditions listed in Lemma 5.3; in particular condition b implies that $L_{\sigma} \cap K^{\perp}$ has constant rank, and condition c is just equation (4.1). Hence, by Proposition 4.1, $L_{\sigma}$ descends to a maximal isotropic subbundle of $\underline{E}$, which by condition b is the image of a splitting $\underline{\sigma}: T \underline{C} \rightarrow \underline{E}$. Notice that if $X \in T C$ is a lift of $\underline{X} \in T \underline{C}$, then $\sigma(X) \in L_{\sigma} \cap K^{\perp}$ is a lift of $\underline{\sigma}(\underline{X}) \in \underline{E}$.

To compute the 3 -form on $\underline{C}$ induced by $j^{*} H_{\sigma}$, pick three tangent vectors on $\underline{C}$ at some point $\underline{p}$, which by abuse by notation we denote by $\underline{X}, \underline{Y}, \underline{Z}$. Extend them to vector fields on $\underline{C}$ and lift them to obtain projectable vector fields $X, Y, Z . \sigma(Z)$ lies in $\Gamma_{\text {bas }}\left(K^{\perp}\right)$, and as seen above it is a lift of $\underline{\sigma}(\underline{Z}) \in \Gamma(\underline{E})$. The same holds for $X$ and $Y$, therefore, by the definition of Courant bracket on $\underline{E}$, we know that $[\sigma(X), \sigma(Y)] \in \Gamma_{\text {bas }}\left(K^{\perp}\right)$ is a lift of $[\underline{\sigma}(\underline{X}), \underline{\sigma}(\underline{Y})] \in \Gamma(\underline{E})$. Hence,

$$
H_{\sigma}(X, Y, Z)=2\langle[\sigma(X), \sigma(Y)], \sigma(Z)\rangle=2\langle[\underline{\sigma}(\underline{X}), \underline{\sigma}(\underline{Y})], \underline{\sigma}(\underline{Z})\rangle .
$$


That is, $H_{\sigma}$ descends to the curvature 3 -form of $\underline{E}$ induced by the isotropic splitting $\underline{\sigma}$.

Remark 5.8. If $\sigma$ and $\hat{\sigma}$ are any two isotropic splittings for $E \rightarrow T M$, then there is a 2-form $b \in \Omega^{2}(M)$ for which $\sigma(X)-\hat{\sigma}(X)=b(X, \cdot) \in T^{*} M$ for all $X \in T M$. It is also known that $H_{\sigma}$ and $H_{\hat{\sigma}}$ differ by $d b$. Now let $\sigma$ and $\hat{\sigma}$ be adapted to $K$; we claim that $j^{*} b$ descends to a 2 -form on $\underline{C}$. Indeed, if $X \in \pi(K), b(X, \cdot)=\sigma(X)-\hat{\sigma}(X) \in K \cap T^{*} M=N^{*} C$, so the interior product of $X$ with $j^{*} b$ vanishes, and the same is true for $d\left(j^{*} b\right)$ as the difference of 3 -forms which descend to $\underline{C}$. This is consistent with the fact that by Proposition 5.7, $H_{\sigma}$ and $H_{\hat{\sigma}}$ descend to 3 -forms that represent the same element of $H^{3}(\underline{C}, \mathbb{R})$ (the Ševera class of $\underline{E}$ ).

As an instance of how a splitting adapted to $K$ is used to compute the Ševera class of the reduced Courant algebroid, we revisit Example 3.12 of [3].

Example 5.9. Let $M=C=S^{3} \times S^{1}$; denote by $\partial_{t}$ the infinitesimal generator of the action of the circle on $S^{3}$ giving rise to the Hopf bundle $p: S^{3} \rightarrow S^{2}$, and by $s$ the coordinate on the second factor $S^{1}$. Let $E=T M \oplus$ $T^{*} M$, the untwisted (i.e., $H=0$ ) Courant algebroid on $M$. We choose the rank 1 subbundle $K$ to be spanned by $\partial_{t}+d s$. Choose a connection one form $\alpha$ for the circle bundle $S^{3} \rightarrow S^{2}$, and denote by $X^{H} \in T S^{3}$ the horizontal lift of a vector $X$ on $S^{2} . K^{\perp}$ is spanned by $\left\{\partial_{t}, \partial_{s}-\alpha, X^{H}, p^{*} \xi, d s\right\}$, where $X$ (resp. $\xi$ ) runs over all vectors (resp. covectors) on $S^{2}$. Since $d s$ is closed, the adjoint action of $\partial_{t}+d s$ is just the Lie derivative w.r.t. $\partial_{t}$, which kills any of $\partial_{t}, \alpha, X^{H}, p^{*} \xi, \partial_{s}, d s$. In particular, $\Gamma_{\text {bas }}\left(K^{\perp}\right)$ spans $K^{\perp}$. Hence, the assumptions of Theorem 3.7 are satisfied, and on $S^{2} \times S^{1}$ we have a reduced exact Courant algebroid. Now we choose the splitting $\sigma: T M \rightarrow K^{\perp}$ as follows:

$$
\sigma\left(\partial_{t}\right)=\partial_{t}+d s, \quad \sigma\left(X^{H}\right)=X^{H}+0 \text { for all } X \in T S^{2}, \quad \sigma\left(\partial_{s}\right)=\partial_{s}-\alpha .
$$

This is a splitting adapted ${ }^{5}$ to $K$.

Now we compute $H_{\sigma}$. If $X, Y$ are vector fields on $S^{2}$, we have $\left[\sigma\left(X^{H}\right), \sigma\left(Y^{H}\right)\right]=\left[X^{H}, Y^{H}\right]+0=\left([X, Y]^{H}-F(X, Y) \partial_{t}\right)+0$, where $F \in \Omega^{2}\left(S^{2}\right)$ is the curvature of $\alpha$. Also $\left[\sigma\left(\partial_{s}\right), \sigma\left(X^{H}\right)\right]=0+p^{*}\left(i_{X} F\right)$, and the analog computation for other other combinations of pairs of $\sigma\left(\partial_{t}\right), \sigma\left(X^{H}\right), \sigma\left(\partial_{s}\right)$ is zero. From this, we deduce that $H_{\sigma}=p^{*} F \wedge d s$, which descends to the 3 -form $F \wedge d s$ on $S^{2} \times S^{1}$, which in turn by Proposition 5.7 represents the Ševera class of $\underline{E}$.

As pointed out in $[\mathbf{3}], F \wedge d s$ defines a non-trivial cohomology class. An "explanation" for this fact is that by Proposition 5.7 to obtain a 3 -form on $C$ that descends to a representative of the Ševera class of $\underline{E}$, we need to choose

\footnotetext{
${ }^{5}$ To check that it maps projectable vector fields to elements of $\Gamma_{\text {bas }}\left(K^{\perp}\right)$, use $\left[\partial_{t}+\right.$ $d s, \cdot]=\mathcal{L}_{\partial_{t}}$.
} 
a splitting adapted to $K$; the trivial splitting $\hat{\sigma}$, which delivers $H_{\hat{\sigma}}=0$, fails to be one because it does not map into $K^{\perp}$.

\section{The case of generalized complex structures}

Let $E$ be an exact Courant algebroid over $M$. Recall that a generalized complex structure is a vector bundle endomorphism $\mathcal{J}$ of $E$ which preserves $\langle\cdot, \cdot\rangle$, squares to $-I d_{E}$ and for which the Nijenhuis tensor

$$
N_{\mathcal{J}}\left(e_{1}, e_{2}\right):=\left[\mathcal{J} e_{1}, \mathcal{J} e_{2}\right]-\left[e_{1}, e_{2}\right]-\mathcal{J}\left(\left[e_{1}, \mathcal{J} e_{2}\right]+\left[\mathcal{J} e_{1}, e_{2}\right]\right)
$$

vanishes.

The following proposition is modeled on Theorem 4.8 of [18].

Proposition 6.1 (Generalized complex reduction). Let $E \rightarrow M$ and $K \rightarrow$ $C$ satisfy the assumptions of Theorem 3.7, so that we have an exact Courant algebroid $\underline{E} \rightarrow \underline{C}$. Let $\mathcal{J}$ be a generalized complex structure on $M$ such that $\mathcal{J} K \cap K^{\perp}$ has constant rank and is contained in $K$. Assume further that $\mathcal{J}$ applied to any basic section of $\mathcal{J} K^{\perp} \cap K^{\perp}$ is again a basic section. Then $\mathcal{J}$ descends to a generalized complex structure $\underline{\mathcal{J}}$ on $\underline{E} \rightarrow \underline{C}$.

Remark 6.2. The linear algebra conditions on $\mathcal{J} K \cap K^{\perp}$ are in particular satisfied when $\mathcal{J} K=K$, in which case $\mathcal{J} K \cap K^{\perp}=K$. The opposite extreme is when $\mathcal{J} K \cap K^{\perp}=\{0\}$.

Proof. First we show that $\underline{J}$ induces a smooth ${ }^{6}$ endomorphism of the vector bundle $K^{\perp} / K$ over $C$. Indeed $\mathcal{J} K \cap K^{\perp} \subset K$ is equivalent to $\mathcal{J} K^{\perp}+K \supset$ $K^{\perp}$, so that $K^{\perp}=K^{\perp} \cap\left(\mathcal{J} K^{\perp}+K\right)=\left(K^{\perp} \cap \mathcal{J} K^{\perp}\right)+K$. From this, it is clear that $K^{\perp} \cap \mathcal{J} K^{\perp}$ maps surjectively under $\Pi: K^{\perp} \rightarrow K^{\perp} / K$. Since $\operatorname{ker}\left(\left.\Pi\right|_{K^{\perp} \cap \mathcal{J} K^{\perp}}\right)=\left(K^{\perp} \cap \mathcal{J} K^{\perp}\right) \cap K=K \cap \mathcal{J} K^{\perp}$, by our constant rank assumption, we obtain a smooth vector bundle $K^{\perp} \cap \mathcal{J} K^{\perp} / \operatorname{ker}\left(\left.\Pi\right|_{K^{\perp} \cap \mathcal{J} K^{\perp}}\right)$ canonically isomorphic to $K^{\perp} / K$.

We use again the assumption $\mathcal{J} K \cap K^{\perp} \subset K$, interpreting it as follows: if $e$ lies in the kernel of $\Pi: K^{\perp} \rightarrow K^{\perp} / K$ and $\mathcal{J} e \in K^{\perp}$, then $\mathcal{J} e$ is still in the kernel. This applies in particular to all $e \in$ $\operatorname{ker}\left(\left.\Pi\right|_{K^{\perp} \cap \mathcal{J} K^{\perp}}\right.$ ) (since $K^{\perp} \cap \mathcal{J} K^{\perp}$ is $\mathcal{J}$-invariant), so we deduce that $\mathcal{J}$ leaves $\operatorname{ker}\left(\Pi_{K^{\perp} \cap \mathcal{J} K^{\perp}}\right)$ invariant, i.e., $\mathcal{J}$ induces a well-defined endomorphism on $K^{\perp} \cap \mathcal{J} K^{\perp} / \operatorname{ker}\left(\left.\Pi\right|_{K^{\perp} \cap \mathcal{J} K^{\perp}}\right) \cong K^{\perp} / K$. Further, it is clear that it squares to -1 and preserves the induced symmetric pairing on $K^{\perp} / K$.

Now take a section $\underline{e}$ of $\underline{E}$, lift it to a (automatically basic) section $e$ of $K^{\perp} \cap \mathcal{J} K^{\perp}$. Then by assumption, $\mathcal{J} e$ is again a basic section; this shows that the endomorphism on $K^{\perp} \cap \mathcal{J} K^{\perp} / \operatorname{ker}\left(\left.\Pi\right|_{K^{\perp} \cap \mathcal{J} K^{\perp}}\right)$ descends to an endomorphism $\mathcal{J}$ of $\underline{E}$.

To show that $\underline{\mathcal{J}}$ is integrable, one can apply the proof of Theorem 6.1 in $[\mathbf{3}]$, where $\mathcal{J}$ is encoded by complex Dirac structures. Alternatively, one can

\footnotetext{
${ }^{6}$ This is clear when $\mathcal{J}$ preserves $K^{\perp}$.
} 
show that the Nijenhuis tensor of $\mathcal{J}$ (which vanishes) restricted to $K^{\perp} \cap \mathcal{J} K^{\perp}$ is a lift $^{7}$ of the Nijenhuis tensor of $\mathcal{J}$.

In Proposition 6.1, the condition that $\mathcal{J}$ preserve $\Gamma_{\text {bas }}\left(K^{\perp} \cap \mathcal{J} K^{\perp}\right)$ does not follow from the integrability of $\mathcal{J}$ (see Example 6.3 below for an explicit example). In Section 7, we will consider submanifolds $C$ for which the integrability of $\mathcal{J}$ does imply all the assumptions of Proposition 6.1, in analogy to the case of coisotropic submanifolds in the Poisson setting.

Example 6.3 (Complex foliations). Take $E$ to be the standard Courant algebroid and $\mathcal{J}$ be given by a complex structure $J$ on $M$. Take $\mathcal{F}$ to be a real integrable distribution on $M$ preserved by $\mathcal{J}$ (so $J$ induces the structure of a complex manifold on each leaf of $\mathcal{F}$ ) and $K=\mathcal{F} \oplus 0$, so that $\underline{M}:=M / \pi(K)=M / \mathcal{F}$ be smooth. The generalized complex structure $\mathcal{J}$ preserves $K$. If $\mathcal{J}$ mapped $\Gamma_{\text {bas }}\left(K^{\perp}\right)$ into itself ${ }^{8}$, then by Proposition 6.1 it would follow that $\underline{M}$ would have an induced generalized complex structure. Further, it would necessarily correspond to an honest complex structure on $\underline{M}$ that makes $M \rightarrow \underline{M}$ into a holomorphic map. However, there are examples for which such a complex structure on $\underline{M}$ does not exist; in [21], Winkelmann quotes an example where $M$ is a twistor space of real dimension 6 and $\underline{M}$ is the 4-dimensional torus.

Example 6.4 (Symplectic foliations). Take again $E$ to be the standard Courant algebroid and $\mathcal{J}$ be given by a symplectic form $\omega$ on $M$. Take $K=\mathcal{F}$ to be a real integrable distribution on $M$. One checks that $\mathcal{J} K \cap K^{\perp}$ is contained in $K$ only if it is trivial, which is equivalent to saying that the leaves of $\mathcal{F}$ are symplectic submanifolds. $\mathcal{J}$ maps basic sections of $\mathcal{J} K^{\perp} \cap K^{\perp}=\mathcal{F}^{\omega} \oplus \mathcal{F}^{\circ}$ into basic sections iff the hamiltonian vector field $X_{\pi^{*} f}$ is a projectable vector field for any function $f$, where pr : $M \rightarrow \underline{M}:=$ $M / \mathcal{F}$. When this is the case, the induced generalized complex structure on $\underline{M}$ is the symplectic structure given by the isomorphism of vector spaces $\mathcal{F}_{x}^{\omega} \cong T_{\operatorname{pr}(x) \underline{M}}($ where $x \in M)$.

Remark 6.5. It is known that a generalized complex manifold $(M, \mathcal{J})$ comes with a canonical Poisson structure $\Pi$, whose sharp map $\sharp$ is given by the composition $T^{*} M \hookrightarrow E \stackrel{\mathcal{J}}{\rightarrow} E \stackrel{\pi}{\rightarrow} T M$. If in Proposition 6.1 we assume that $\mathcal{J}$ preserves $K$, then $C$ is a necessarily a coisotropic submanifold, because from $N^{*} C=\left(\pi\left(K^{\perp}\right)\right)^{\circ}=K \cap \operatorname{ker}(\pi) \subset K$, we have $\sharp\left(N^{*} C\right)=\pi\left(\mathcal{J} N^{*} C\right) \subset \pi(K) \subset \pi\left(K^{\perp}\right)=T C$. So $C / \sharp N^{*} C$ (if smooth) has an induced Poisson structure. We know that also $\underline{C}:=C / \pi(K)$ has a

\footnotetext{
${ }^{7}$ The computation is straightforward except for showing that the term $\left[e_{1}, \mathcal{J} e_{2}\right]+$ $\left[\mathcal{J} e_{1}, e_{2}\right]$ of $(6.1)$ is a section of $\mathcal{J} K^{\perp}$ for all $e_{1}, e_{2} \in \Gamma_{\text {bas }}\left(K^{\perp} \cap \mathcal{J} K^{\perp}\right)$, which follows using the Leibniz rule $\mathrm{C} 4$ ).

${ }^{8}$ This is equivalent to saying that for any vector field $X$ on $M$ which is projectable the vector field $J(X)$ is also projectable.
} 
Poisson structure, induced from the reduced generalized complex structure. In general, $\pi(K)$ is not the characteristic distribution of $C$; we just have an inclusion $\sharp N^{*} C \subset \pi(K)^{9}$. The projection $C / \sharp N^{*} C \rightarrow C / \pi(K)$ is a Poisson map.

Given an exact Courant algebroid $E$ on $M$, recall that a generalized Kähler structure consists of two commuting generalized complex structures $\mathcal{J}_{1}, \mathcal{J}_{2}$ such that the symmetric bilinear form on $E$ given by $\left\langle\mathcal{J}_{1} \mathcal{J}_{2} \cdot, \cdot\right\rangle$ be positive definite. The following result borrows the proof of Theorem 6.1 of $[\mathbf{3}]$.

Proposition 6.6 (Generalized Kähler reduction). Let $E \rightarrow M$ and $K \rightarrow C$ satisfy the assumptions of Proposition 3.7, so that we have an exact Courant algebroid $\underline{E} \rightarrow \underline{C}$. Let $\mathcal{J}_{1}, \mathcal{J}_{2}$ be a generalized Kähler structure on $M$ such that $\mathcal{J}_{1} K=K$. Assume further that $\mathcal{J}_{1}$ maps $\Gamma_{\text {bas }}\left(K^{\perp}\right)$ into itself and that $\mathcal{J}_{2}$ maps $\Gamma_{\text {bas }}\left(\mathcal{J}_{2} K^{\perp} \cap K^{\perp}\right)$ into itself. Then $\mathcal{J}_{1}, \mathcal{J}_{2}$ descend to a generalized Kähler structure on $\underline{E} \rightarrow \underline{C}$.

Proof. By Proposition 6.1, $\mathcal{J}_{1}$ induces a generalized complex structure $\underline{\mathcal{J}_{1}}$ on $\underline{E}$. The orthogonal $K^{\mathcal{G}}$ of $K$ w.r.t. $\left\langle\mathcal{J}_{1} \mathcal{J}_{2}, \cdot\right\rangle$ is $\left(\mathcal{J}_{2} \mathcal{J}_{1} K\right)^{\perp}=\mathcal{J}_{2} K^{\perp}$. Because of the identity $K^{\perp}=K \oplus\left(K^{\mathcal{G}} \cap K^{\perp}\right)$, the restriction to $\mathcal{J}_{2} K^{\perp} \cap$ $K^{\perp}$ of the projection $K^{\perp} \rightarrow K^{\perp} / K$ is an isomorphism. So we can apply Proposition 6.1 to $\mathcal{J}_{2}$ and obtain a generalized complex structure $\underline{\mathcal{J}_{2}}$ on $\underline{E}$. Notice that both $\mathcal{J}_{1}$ and $\mathcal{J}_{2}$ preserve $\mathcal{J}_{2} K^{\perp} \cap K^{\perp}$; pulling back sections of $\underline{E}$ to basic sections of $\mathcal{J}_{2} K^{\perp} \cap K^{\perp}$, one sees that $\mathcal{J}_{1}, \mathcal{J}_{2}$ form a generalized Kähler structure on $\underline{E}$.

\section{The case of (weak) branes}

In this section, we define branes and show that they admit a natural quotient which is a generalized complex manifold endowed with a space-filling brane. Then we notice that quotients of more general objects, which we call "weak branes", also inherit a generalized complex structure; examples of weak branes are coisotropic submanifolds in symplectic manifolds. Finally we show how weak branes can be obtained by passing from a generalized complex manifold to a suitable submanifold.

\subsection{Reducing branes.}

Definition 7.1. Let $E$ be an exact Courant algebroid over a manifold $M$. A generalized submanifold is a pair $(C, L)$ consisting of a submanifold $C \subset M$ and a maximal isotropic subbundle $L \subset E$ over $C$ with $\pi(L)=T C$, which is closed under the Courant bracket (i.e., $[\Gamma(L), \Gamma(L)] \subset \Gamma(L)$ with the conventions of Remark 3.2).

\footnotetext{
${ }^{9}$ A case in which this inclusion is strict is when $\mathcal{J}$ corresponds to the standard complex structure on $M=\mathbb{C}^{n}$ (with complex coordinates $\left.z_{k}=x_{k}+i y_{k}\right)$ and $K=\operatorname{span}\left\{\frac{\partial}{\partial x_{1}}, \frac{\partial}{\partial y_{1}}\right\}$.
} 
This definition, which already appeared in the literature ${ }^{10}$, is just a splitting-independent rephrasing ${ }^{11}$ of Gualtieri's original definition (Definition 7.4 of $[\mathbf{7}]$ ); see also Lemma 3.2 .3 of [12].

Lemma 7.2. Let $E$ be an exact Courant algebroid over $M$. Choose an isotropic splitting $\sigma$ for $E$, giving rise to an isomorphism of Courant algebroids $(E,[\cdot, \cdot]) \cong\left(T M \oplus T^{*} M,[\cdot, \cdot]_{H_{\sigma}}\right)$, where $H_{\sigma}$ is the curvature 3-form of the splitting. Then pairs $(C, L)$ as in Definition 7.1 correspond bijectively to pairs $(C, F)$, where $F \in \Omega^{2}(C)$ satisfies $-i^{*} H_{\sigma}=d F$ (for $i$ the inclusion of $C$ in $M)$.

Proof. The fact that $L \subset E$ is maximal isotropic and $\pi(L)=T C$ means that under the isomorphism it maps to

$$
\tau_{C}^{F}:=\left\{\left.(X, \xi) \in T C \oplus T^{*} M\right|_{C}:\left.\xi\right|_{T C}=i_{X} F\right\}
$$

for some 2-form $F$ on $C$. The correspondence $L \leftrightarrow F$ is clearly bijective. The integrability conditions correspond because of the following fact, which follows from a straight-forward computation: if $X_{i}+\xi_{i}$ are sections of $\tau_{C}^{F}$, then

$$
2\left\langle\left[X_{1}+\xi_{1}, X_{2}+\xi_{2}\right], X_{3}+\xi_{3}\right\rangle=\left(i^{*} H+d F\right)\left(X_{1}, X_{2}, X_{3}\right) .
$$

By Lemma 7.2, the following definition is equivalent to Gualtieri's original one (i.e., to Definition 7.6 of [7], again up to a sign):

Definition 7.3. Let $E$ be an exact Courant algebroid over a manifold $M$ and $\mathcal{J}$ be a generalized complex structure on $E$. A generalized complex submanifold or brane is a generalized submanifold $(C, L)$ satisfying $\mathcal{J}(L)=L$.

Now we state the main theorem of this paper. Recall that we gave the definition of coisotropic submanifold in Remark 6.5.

Theorem 7.4 (Brane reduction). Let $E$ be an exact Courant algebroid over a manifold $M, \mathcal{J}$ a generalized complex structure on $E$, and $(C, L)$ a brane. Then $C$ is coisotropic w.r.t. the Poisson structure induced by $\mathcal{J}$ on $M$. If the quotient $\underline{C}$ of $C$ by its characteristic foliation is smooth, then

a) $E$ induces an exact Courant algebroid $\underline{E}$ over $\underline{C}$;

b) $\mathcal{J}$ induces a generalized complex structure $\underline{\mathcal{J}}$ on $\underline{E} \rightarrow \underline{C}$;

c) L induces the structures of a space-filling brane on $\underline{C}$ and the Ševera class of $\underline{E}$ is trivial.

\footnotetext{
${ }^{10}$ It appeared in Definition 3.2.2 of $[\mathbf{1 2}]$ with the name "maximally isotropic extended submanifold". Also, a subbundle $L$, as above, but for which we just ask $\pi(L) \subset T C$ is called generalized Dirac structure in Definition 6.8 of [1] (in the setting of the skewsymmetric Courant bracket).

${ }^{11} \mathrm{Up}$ to a sign, since Definition 7.4 of $[7]$ requires $i^{*} H_{\sigma}=d F$ (in the notation of this lemma).
} 
Proof. Recall that the Poisson structure $\Pi$ induced by $\mathcal{J}$ on $M$ (or rather its sharp map $\sharp)$ is given by the composition $T^{*} M \hookrightarrow E \stackrel{\mathcal{J}}{\rightarrow} E \stackrel{\pi}{\rightarrow} T M$. Since $N^{*} C=(\pi(L))^{\circ}=L \cap \operatorname{ker}(\pi) \subset L$, we have $\sharp\left(N^{*} C\right)=\pi\left(\mathcal{J} N^{*} C\right) \subset \pi(L)=$ $T C$, so $C$ is a coisotropic submanifold. Let $\mathcal{F}:=\sharp N^{*} C$.

a) $C, L$, and $\mathcal{F}$ satisfy the assumptions of Proposition 5.6, hence we obtain an exact Courant algebroid $\underline{E}$ over $\underline{C}$ (constructed as in Theorem 3.7 with $\left.K:=L \cap \pi^{-1}(\mathcal{F})\right)$. Notice that we have not made use of the integrability of $\mathcal{J}$ here, if not for the fact that the induced bivector $\Pi$ is integrable and hence the distribution $\mathcal{F}$ is involutive.

b) Now we check that the assumptions of Proposition 6.1 are satisfied. From $L \cap T^{*} M=N^{*} C$, the fact that $\mathcal{J} N^{*} C$ is contained in $L$, and that it projects onto $\mathcal{F}$, we deduce that $K=N^{*} C+\mathcal{J} N^{*} C$, which is clearly preserved by $\mathcal{J}$. So we just need to check that, for any basic section $e$ of $K^{\perp}$, $\mathcal{J} e$ is again basic. Locally we can write $K=\operatorname{span}\left\{\left.\left(d g_{i}\right)\right|_{C},\left.\mathcal{J}\left(d g_{i}\right)\right|_{C}\right\}$, where $g_{1}, \ldots, g_{\text {codim }(C)}$ are local functions on $M$ vanishing on $C$. Since each $d g_{i}$ is a closed 1-form, $\left[\left.\left(d g_{i}\right)\right|_{C}, \mathcal{J} e\right] \subset K$. Using the fact that the Nijenhuis tensor $N_{\mathcal{J}}$ vanishes (6.1), we have

$$
\left[\left.\mathcal{J}\left(d g_{i}\right)\right|_{C}, \mathcal{J} e\right]=\mathcal{J}\left[\left.\mathcal{J}\left(d g_{i}\right)\right|_{C}, e\right]+\mathcal{J}\left[\left.\left(d g_{i}\right)\right|_{C}, \mathcal{J} e\right]+\left[\left.\left(d g_{i}\right)\right|_{C}, e\right] .
$$

The first term on the r.h.s. lies in $K$ because $e$ is a basic section, and the last two because $d g_{i}$ is a closed 1-form. So $\left[\left.\mathcal{J}\left(d g_{i}\right)\right|_{C}, \mathcal{J} e\right] \subset K$, hence $e$ is again a basic section. Hence, the assumptions of Proposition 6.1 are satisfied, concluding the proof of $b$.

c) We want to apply Proposition 4.1 to obtain a brane on $\underline{C}$. Since $L \subset K^{\perp}$, the assumption (4.1) needed for $L$ to descend reads $[\Gamma(K), \Gamma(L)] \subset \Gamma(L)$, and the integrability assumption (4.2) reads $\left[\Gamma_{\text {bas }}(L), \Gamma_{\text {bas }}(L)\right] \subset \Gamma(L)$. As $L$ is closed under the bracket, both assumptions hold, and we obtain an (integrable) Dirac structure $\underline{L}$ on $\underline{C}$. Furthermore, from the fact that $\mathcal{J}$ preserves $L$, we see that $\underline{\mathcal{J}}$ preserves $\underline{L}$. Hence, $(\underline{C}, \underline{L})$ is a brane for the generalized complex structure $\underline{\mathcal{J}}$ on $\underline{E}$.

If we choose any isotropic splitting for $\underline{E}$, as discussed in Lemma 7.2, then $\underline{L}$ gives rise to a 2 -form $\hat{F}$ on $\underline{C}$ such that $-d \hat{F}$ equals the curvature of the splitting, which hence is an exact 3 -form. This concludes the proof of c and of the theorem.

Remark 7.5. Let us denote with $(\underline{C}, \underline{\mathcal{J}})$ a generalized complex manifold admitting a space-filling brane $(\underline{C}, \underline{L})$. By Example 6.12 of [8], using the splitting $T \underline{C} \rightarrow \underline{E}$ with image $\underline{L}$, the exact Courant algebroid $\underline{E}$ is identified with the untwisted Courant algebroid, and the generalized complex structure assumes the form

$$
\left(\begin{array}{cc}
-\underline{I} & \underline{\Pi} \\
0 & \underline{I}^{*}
\end{array}\right)
$$


Here $\underline{I}$ is an honest complex structure on $\underline{C}$, which corresponds to $\underline{J}: \underline{L} \rightarrow \underline{L}$ under the identification $\underline{L} \cong T \underline{C}$ given by the anchor, and $\underline{\Pi}$ is the Poisson structure induced by $\mathcal{J}$.

Further, $\underline{\Pi}$ is the imaginary part of a holomorphic Poisson bivector field on $\underline{C}$. Therefore, Theorem 7.4 implies that the generalized complex structure $\mathcal{J}$ on the quotient of a brane (when smooth) is a holomorphic Poisson deformation of a complex structure.

Now assume the set-up of Theorem 7.4, i.e., that $E$ is an exact Courant algebroid over $M, \mathcal{J}$ a generalized complex structure, and $(C, L)$ a brane with smooth quotient $\underline{C}$. Encoding generalized complex structures by their $+i$-eigenbundles Gualtieri (Corollary 6.6 of $[\mathbf{8}]$ ) provides a direct way to describe the reduced generalized complex structure $\mathcal{J}$ we obtained in Theorem 7.4, as follows. We saw that in a suitable splitting, the reduced generalized complex structure is given by (7.2), hence it is determined by the tensors $\underline{I}$ and $\underline{\Pi}$ appearing there. Let $\ell$ denote the $+i$-eigenbundle of $J: L \otimes \mathbb{C} \rightarrow L \otimes \mathbb{C}$, and $A:=\pi(\ell) \subset T C \otimes \mathbb{C}$. Then $A$ descends to a distribution $\underline{A}$ on $\underline{C}$ so that $\underline{A} \oplus \underline{A}=T \underline{C} \otimes \mathbb{C}$, which defines a complex structure on $\underline{C}$. This agrees with the complex structure $\underline{I}$, since the $+i$-eigenbundle of the complexification of $\underline{I}$ is the image under $\underline{\pi}$ of the $+i$ eigenbundle of $\mathcal{J}: \underline{L} \otimes \mathbb{C} \rightarrow \underline{L} \otimes \mathbb{C}$, which is just $\pi(\ell)=\underline{A}$. The Poisson structure $\underline{\Pi}$ can be obtained directly simply by coisotropic reduction.

Remark 7.6. We saw in Theorem 7.4 that branes $C$ are coisotropic and their quotient by the characteristic foliation is endowed with a generalized complex structure. As pointed out in Remark 6.5, if one starts with a $\mathcal{J}$ invariant coisotropic subbundle $K^{\perp}$ of $\left.E\right|_{C}$ (instead of constructing one from the brane $(C, L)$ as in Theorem 7.4) in general, it is a different quotient of $C$ that is endowed with a generalized complex structure (via Proposition 6.1). If one picks just any arbitrary coisotropic submanifold $C$, its quotient by the characteristic foliation inherits a Poisson structure, but in general it does not inherit a generalized complex structure: take for example any odd dimensional submanifold of a complex manifold.

Remark 7.7. When the characteristic foliation of a brane $(C, L) \subset M$ is regular, using coordinates adapted to the foliation, one sees that the quotient of small enough open sets $U$ of $C$ by the characteristic foliation is smooth, and Theorem 7.4 gives a local statement. However, in general, the characteristic foliation is singular, as the following example shows. Take $M=\mathbb{C}^{2}$, the untwisted exact Courant algebroid as $E$, and as $\mathcal{J}$ take $\left(\begin{array}{cc}I & \Pi \\ 0 & -I^{*}\end{array}\right)$. Here $I\left(\partial_{x_{i}}\right)=\partial_{y_{i}}$ is the canonical complex structure on $\mathbb{C}^{2}$ and $\Pi=y_{1}\left(\partial_{x_{1}} \wedge\right.$ $\left.\partial_{x_{2}}-\partial_{y_{1}} \wedge \partial_{y_{2}}\right)-x_{1}\left(\partial_{y_{1}} \wedge \partial_{x_{2}}+\partial_{x_{1}} \wedge \partial_{y_{2}}\right)$ is the imaginary part of the holomorphic Poisson bivector (see $[\mathbf{8}, \mathbf{9}]) z_{1} \partial_{z_{1}} \wedge \partial_{z_{2}}$. It is easy to check that $C=\left\{z_{2}=0\right\}$ with $F=0$ define a brane for $\mathcal{J}$, and that the characteristic distribution of $C$ has rank zero at the origin and rank 2 elsewhere. 
Example 7.8 (Branes in symplectic manifolds). Consider a symplectic manifold $(M, \omega)$ and view it as a generalized complex structure $\mathcal{J}=$ $\left(\begin{array}{cc}0 & -\omega^{-1} \\ \omega & 0\end{array}\right)$ on the standard Courant algebroid. Let $(C, F)$ be a brane, i.e., $F$ is a closed 2-form on $C$ such that $L:=\tau_{C}^{F}$ is preserved by $\mathcal{J} . F$ descends to the quotient of $C$ by the characteristic distribution $\mathcal{F}:=T C^{\omega}$, hence the isotropic subbundle $K=L \cap \pi^{-1}(\mathcal{F})$ defined in the proof of Theorem 7.4a is just $\mathcal{F} \oplus N^{*} C$. The reduced Courant algebroid $K^{\perp} / K$ is therefore canonically isomorphic to $T \underline{C} \oplus T^{*} \underline{C}$, and the reduced generalized complex structure is $\underline{\mathcal{J}}=\left(\begin{array}{cc}0 & -\underline{\omega}^{-1} \\ \underline{\omega} & 0\end{array}\right)$, where $\underline{\omega}$ denotes the symplectic form on $\underline{C}$ obtained from $\omega$.

The Dirac structure on $\underline{C}$ obtained in Theorem 7.4c pushing forward $L$ is just $\operatorname{graph}(\underline{F})$, where $\underline{F} \in \Omega^{2}(\underline{C})$ denotes the pushforward of $F \in \Omega^{2}(C)$. The action of $\underline{\mathcal{J}}$ on graph $(\underline{F})$ delivers $\underline{I}:=-\underline{\omega}^{-1} \underline{F}$ for the complex structure on $\underline{C}$ induced as in Remark 7.5. Applying the gauge transformation by the closed 2-form $\underline{F}$ brings $\mathcal{J}$ into the form (7.2) (with $\underline{\Pi}=-\underline{\omega}^{-1}$ ).

Further, as shown in Example 7.8 of $[\mathbf{7}], \underline{F}+i \underline{\omega}$ is a holomorphic symplectic form on $\underline{C}$.

Remark 7.9. Suppose that in the setting of Theorem $7.4 E$ is additionally endowed with some $\mathcal{J}_{2}$ so that $\mathcal{J}_{1}, \mathcal{J}_{2}$ form a generalized Kähler structure. Then using Proposition 6.6, we see that if $\mathcal{J}_{2}$ descends to $\underline{E}$, then $\underline{E}$ is endowed with a generalized Kähler structure too.

7.2. Reducing weak branes. We weaken the conditions in the definition of brane; at least for the time being, we refer to resulting object as "weak branes".

Definition 7.10. Let $E$ be an exact Courant algebroid over a manifold $M$, $\mathcal{J}$ a generalized complex structure on $E$. We will call weak brane a pair $(C, L)$ consisting of a submanifold $C$ and a maximal isotropic subbundle $\left.L \subset E\right|_{C}$ with $\pi(L)=T C$ such that

$$
\mathcal{J}\left(N^{*} C\right) \subset L, \quad[\Gamma(K), \Gamma(L)] \subset \Gamma(L)
$$

(where $K:=L \cap \pi^{-1}(\mathcal{F})$ and $\mathcal{F}:=\sharp N^{*} C$, or equivalently $K=N^{*} C+$ $\left.\mathcal{J} N^{*} C\right)$.

Notice that weak branes for which $\mathcal{F}$ has constant rank automatically satisfy the assumptions of Proposition 5.6. Also notice that in the proof of Theorem 7.4 (except for c), we just used properties of weak branes, hence we obtain

Proposition 7.11. Assume the setup of Theorem 7.4 but allowing $(C, L)$ to be a weak brane. Then $a$ and $b$ of Theorem 7.4 still hold, i.e., there is a reduced Courant algebroid and a reduced generalized complex structure on $\underline{C}$. 
Remark 7.12. The reduced Courant algebroid and generalized complex structure depend on $C$ but not on $L$, since they are constructed out of the subbundle $K=N^{*} C+\mathcal{J} N^{*} C$.

We describe how weak branes look like in the split case, i.e., when $E=$ $\left(T M \oplus T^{*} M,[\cdot, \cdot]_{H}\right)$. We write $\mathcal{J}$ in matrix form as $\left(\begin{array}{cc}A & \Pi \\ \omega & -A^{*}\end{array}\right)$, where $A$ is an endomorphism of $T M, \Pi$ the Poisson bivector canonically associated to $\mathcal{J}$, and $\omega$ a 2 -form on $M$.

Corollary 7.13. Let $C$ be a submanifold of $M$ and $F \in \Omega^{2}(C)$. Fix an extension $B \in \Omega^{2}(M)$ of $F$. Then $\left(C, \tau_{C}^{F}\right)$ is a weak brane (with smooth quotient $\underline{C}$ ) iff $C$ is coisotropic (with smooth quotient $\underline{C}$ ), $A+\Pi B: T M \rightarrow$ $T M$ preserves $T C$, and the 3-form $d F+i^{*} H$ on $C$ descends to $\underline{C}$.

In this case, the Ševera class of the reduced Courant algebroid $\underline{E}$ is represented by the pushforward of $d F+i^{*} H$. Further, there is a splitting of $\underline{E}$ in which the reduced generalized complex structure is

$$
\underline{\tilde{\mathcal{J}}}=\left(\begin{array}{cc}
\frac{\tilde{\tilde{A}}}{\tilde{\tilde{\omega}}} & \underline{\tilde{\tilde{\Pi}}} \\
\underline{\tilde{A}^{*}}
\end{array}\right)
$$

where the endomorphism $\underline{\tilde{A}}$ is the pushforward of $\left.(A+\Pi B)\right|_{T C}$, the Poisson bivector $\underline{\underline{\Pi}}$ is induced by $\bar{\Pi}$, and the 2-form $\underline{\tilde{\omega}}$ is the pushforward of $i^{*}(\omega-$ $\left.B \Pi B-B A-A^{*} B\right)$.

Proof. Since $K$ is $\tau_{C}^{F} \cap \pi^{-1}(\mathcal{F})$, equation (7.1) shows that $\left[\Gamma(K), \Gamma\left(\tau_{C}^{F}\right)\right] \subset$ $\Gamma\left(\tau_{C}^{F}\right)$ is equivalent to the fact that the closed 3 -form $i^{*} H+d F$ descend to $\underline{C}$. Now perform a $-B$-transformation; the transformed objects are $\tilde{L}=$ $T C \oplus N^{*} C$ and $\tilde{\mathcal{J}}=\left(\begin{array}{cc}\tilde{A} & \tilde{\Pi} \\ \tilde{\omega} & -\tilde{A}^{*}\end{array}\right)$, with components $\tilde{A}=A+\Pi B, \tilde{\Pi}=\Pi$, and $\tilde{\omega}=\omega-B \Pi B-B A-A^{*} B$ (see, for example, $[\mathbf{2 0}]$ ). Hence, we see that the first condition in (7.3) is equivalent to $C$ begin coisotropic and $A+\Pi B$ preserving $T C$ (a condition independent of the extension $B$ ). Further, since by the proof of Theorem $7.4 \mathcal{J}$ preserves $T C \oplus \mathcal{F}^{\circ}$ and $\mathcal{F} \oplus N^{*} C$, it is clear that in the induced splitting of $\underline{E}$, the components of $\underline{\mathcal{J}}$ are induced from those of $\tilde{\mathcal{J}}$.

Now we show that the Ševera class of the reduced Courant algebroid $\underline{E}$ is represented by the pushforward of $d F+i^{*} H$. By the proof of Lemma 5.4, any isotropic splitting $\sigma$ of $\left(T M \oplus T^{*} M,[\cdot, \cdot]_{H}\right)$ with $\sigma(T C) \subset \tau_{C}^{F}$ (for example, one is given by $\sigma(X):=X+i_{X} B$ ) is automatically a splitting adapted to $K$. Hence, by Proposition $5.7, i^{*} H_{\sigma}$ pushes down to a representative of the Ševera class of $\underline{E}$. Now $i^{*} H_{\sigma}$ is just $d F+i^{*} H$, because for vectors $X_{i} \in T C$ we have $H_{\sigma}\left(X_{1}, X_{2}, X_{3}\right)=2\left\langle\left[\sigma\left(X_{1}\right), \sigma\left(X_{2}\right)\right], \sigma\left(X_{3}\right)\right\rangle=$ $\left(i^{*} H+d F\right)\left(X_{1}, X_{2}, X_{3}\right)$, where we used $\sigma\left(X_{i}\right) \subset \tau_{C}^{F}$ and (7.1) in the last equality.

Example 7.14 (Coisotropic reduction). If $\mathcal{J}$ corresponds to a symplectic structure on $M$, then any coisotropic submanifold $C$ endowed with $F=0$ 
is a weak brane. The generalized complex structure on $\underline{C}$ (assumed to be a smooth manifold) corresponds to the reduced symplectic form.

If $\mathcal{J}$ corresponds to a complex structure, then any weak brane is necessarily a complex submanifold. If $\mathcal{J}$ is obtained deforming a complex structure in direction of a holomorphic Poisson structure $[\mathbf{8}, \mathbf{9}]$, this is no longer the case, as in the following two examples. In both cases, however, the reduced generalized complex structures we obtain are quite trivial.

Example 7.15. Similarly to Remark 7.7 take $M$ to be the open halfspace $\left\{\left(x_{1}, y_{1}, x_{2}, y_{2}\right): y_{1}>0\right\} \subset \mathbb{C}^{2}$, the untwisted exact Courant algebroid as $E$, and as $\mathcal{J}$ take $\left(\begin{array}{cc}I & \Pi_{1} \\ 0 & -I^{*}\end{array}\right)$, where $I\left(\partial_{x_{i}}\right)=\partial_{y_{i}}$ is the canonical complex structure on $\mathbb{C}^{2}$ and $\Pi=y_{1}\left(\partial_{x_{1}} \wedge \partial_{x_{2}}-\partial_{y_{1}} \wedge \partial_{y_{2}}\right)-x_{1}\left(\partial_{y_{1}} \wedge \partial_{x_{2}}+\partial_{x_{1}} \wedge \partial_{y_{2}}\right)$ is the imaginary part of the holomorphic Poisson bivector $z_{1} \partial_{z_{1}} \wedge \partial_{z_{2}}$. We now take $C=\left\{\left(x_{1}, y_{1}, x_{2}, 0\right): y_{1}>0\right\}$ and on $C$ the closed 2-form $F:=-\frac{1}{y_{1}} d y_{1} \wedge d x_{2}$. We show that the pair $(C, F)$ forms a weak brane. By dimension reasons, $C$ is coisotropic (the characteristic distribution is regular and spanned by $\left.x_{1} \partial_{x_{1}}+y_{1} \partial_{y_{1}}\right)$, so we just have to check that $I+\Pi B$ preserves $T C$, where $B$ is the 2 -form on $M$ given by the same formula as $F$. This is true as one computes $I+\Pi B: \partial_{x_{1}} \mapsto \partial_{y_{1}}, \partial_{y_{1}} \mapsto-\frac{x_{1}}{y_{1}} \partial_{y_{1}}, \quad \partial_{x_{2}} \mapsto-\frac{x_{1}}{y_{1}} \partial_{x_{2}}$.

Now we want to compute the generalized complex structure on $\underline{C}$ given by Proposition 7.11, We do so by first applying the gauge transformation by $-B$ to obtain a generalized complex structure $\tilde{\mathcal{J}}$ and then using the diffeomorphism $\underline{C} \cong\left(-\frac{\pi}{2}, \frac{\pi}{2}\right) \times \mathbb{R}$ induced by $C \rightarrow\left(-\frac{\pi}{2}, \frac{\pi}{2}\right) \times \mathbb{R},\left(x_{1}, y_{1}, x_{2}\right) \mapsto$ $\left(\theta:=\operatorname{arctg}\left(\frac{x_{1}}{y_{1}}\right), x_{2}\right)$. The Poisson bracket of the coordinate functions $\theta$ and $x_{2}$ on $\underline{C}$ is computed by pulling back the two functions to $C$, extending them to the whole of $M$ and taking their Poisson bracket there. This gives the constant function 1 . Next the coordinate vector field $\partial_{\theta}$ on $\underline{C}$ is lifted by the vector field $\frac{x_{1}^{2}+y_{1}^{2}}{y_{1}} \partial_{x_{1}}$ on $C$, and of course $\partial_{x_{2}}$ on $\underline{C}$ is lifted by $\partial_{x_{2}}$ on $C$. Applying the endomorphism $I+\Pi B$ of $T C$, we see the induced endomorphism on $T \underline{C}$ is just multiplication by $-t g(\theta)$. Finally, the component $\tilde{\omega}$ of $\tilde{\mathcal{J}}$ is given by $-B I-B \Pi B-I^{*} B$, which on $C$ restricts to the 2-form $\frac{1}{y_{1}^{2}}\left(y_{1} d x_{1}-x_{1} d y_{1}\right) \wedge d x_{2}$, which in turn is the pullback of the 2 -form $\left(1+\operatorname{tg}^{2}(\theta)\right) d \theta \wedge d x_{2}$ on $\underline{C}$. Hence, the induced generalized complex structure on $\underline{C}$ is

$$
\left(\begin{array}{cc}
-t g(\theta) \cdot I d & \partial_{\theta} \wedge \partial_{x_{2}} \\
\left(1+t g^{2}(\theta)\right) d \theta \wedge d x_{2} & t g(\theta) \cdot I d
\end{array}\right) .
$$

This is just the gauge transformation by the closed 2 -form $\operatorname{tg}(\theta) d \theta \wedge d x_{2}$ of the generalized complex structure on $\left(-\frac{\pi}{2}, \frac{\pi}{2}\right) \times \mathbb{R}$ that corresponds to the symplectic form $d \theta \wedge d x_{2}$.

Example 7.16. Similarly to the previous example we take $M=\mathbb{C}^{2}$, the untwisted exact Courant algebroid as $E$, and as $\mathcal{J}$ we take $\left(\begin{array}{cc}I & \Pi_{1} \\ 0 & -I^{*}\end{array}\right)$, where 
$I\left(\partial_{x_{i}}\right)=\partial_{y_{i}}$ is the canonical complex structure on $\mathbb{C}^{2}$ and $\Pi=y_{1}\left(\partial_{x_{1}} \wedge\right.$ $\left.\partial_{x_{2}}-\partial_{y_{1}} \wedge \partial_{y_{2}}\right)-x_{1}\left(\partial_{y_{1}} \wedge \partial_{x_{2}}+\partial_{x_{1}} \wedge \partial_{y_{2}}\right)$. Now we let $C$ be the hypersurface $\left\{x_{1}^{2}+y_{1}^{2}=1\right\}$. The characteristic distribution is generated by $\partial_{y_{2}}$, so the quotient $\underline{C}$ is a cylinder. Let $a, b, c \in C^{\infty}(C)$ so that, denoting by $F_{(a, b, c)}$ the pullback to $C$ of

$$
\begin{aligned}
B_{(a, b, c)}:= & a \cdot d x_{1} \wedge d y_{1}+b \cdot d x_{1} \wedge d x_{2}+c \cdot d y_{1} \wedge d x_{2}-y_{1} \cdot d x_{1} \wedge d y_{2} \\
& +x_{1} \cdot d y_{1} \wedge d y_{2},
\end{aligned}
$$

$d F_{(a, b, c)}$ descends $^{12}$ to $\underline{C}$. One checks that $I^{*}+B_{(a, b, c)} \Pi$ preserves $N^{*} C$, so that $\left(C, F_{(a, b, c)}\right)$ is a weak brane. A computation analog to the one of the previous example shows that the reduced generalized complex structure on $\underline{C}=S^{1} \times \mathbb{R}$ with coordinates $\theta$ and $x_{2}$ is

$$
\left(\begin{array}{cc}
\lambda_{(a, b)} \cdot I d & \partial_{\theta} \wedge \partial_{x_{2}} \\
\left(1+\lambda_{(a, b)}^{2}\right) d \theta \wedge d x_{2} & -\lambda_{(a, b)} \cdot I d
\end{array}\right)
$$

where $\lambda_{(a, b)} \in C^{\infty}(\underline{C})$ is the function that lifts to $-b y_{1}+c x_{1} \in C^{\infty}(C)$ via $C \rightarrow \underline{C}$. Again this is a gauge transformation of the standard symplectic structure on $S^{1} \times \mathbb{R}$.

A consequence is that for no choice of $a, b, c$ as above, the weak brane $\left(C, F_{(a, b, c)}\right)$ is actually a brane. Indeed if this was the case by Theorem 7.4, we would obtain a space-filling brane for a symplectic structure on $S^{1} \times \mathbb{R}$; applying again Theorem 7.4, by Example 7.8 of [7], we would obtain the structure of a holomorphic symplectic manifold on $S^{1} \times \mathbb{R}$, which cannot exist because holomorphic symplectic manifolds have real dimension $4 k$.

7.3. Cosymplectic submanifolds. Recall that a submanifold $\tilde{M}$ of a Poisson manifold $(M, \Pi)$ is cosymplectic if $\sharp N^{*} \tilde{M} \oplus T \tilde{M}=\left.T M\right|_{\tilde{M}}$. It is known (see, for example, [22]) that a cosymplectic submanifold inherits canonically a Poisson structure. The following lemma, which follows also from more general results of [2], says that generalized complex structures are also inherited by cosymplectic submanifolds.

Lemma 7.17. Let $E$ be an exact Courant algebroid over a manifold $M, \mathcal{J}$ a generalized complex structure on $E$, and $\tilde{M}$ a cosymplectic submanifold of $M$ (w.r.t. the natural Poisson structure on $M$ induced by $\mathcal{J})$. Then $\tilde{M}$ is naturally endowed with a generalized complex structure.

Proof. We want to apply Proposition 6.1 with $K=N^{*} \tilde{M}$ (so $K^{\perp}=$ $\left.\pi^{-1}(T \tilde{M})\right)$. The intersection $\mathcal{J} K \cap K^{\perp}$ is trivial. Indeed if $\xi \in N^{*} \tilde{M}$ and $\pi(\mathcal{J} \xi) \in T \tilde{M}$, then by the definition of cosymplectic submanifold, $\pi(\mathcal{J} \xi)=0$ (recall that $\sharp=\left.\pi \mathcal{J}\right|_{T^{*} M}$ ) and the restriction $\sharp$ to $N^{*} \tilde{M}$ is injective, so that $\xi=0$. Further, all sections of $K^{\perp}$ are basic, so $\mathcal{J}$ maps the set of basic

\footnotetext{
${ }^{12}$ This happens exactly when $F_{(a, b, c)}$ is closed.
} 
sections of $\mathcal{J} K^{\perp} \cap K^{\perp}$ into itself. Hence, the assumptions of Proposition 6.1 are satisfied and we obtain a generalized complex structure on $\tilde{M}$.

Now we describe how a pair $(C, L)$ which does not quite satisfy the conditions of Definition 7.10 can be regarded as a weak brane by passing to a cosymplectic submanifold.

Proposition 7.18. Let $E$ be an exact Courant algebroid over a manifold $M, \mathcal{J}$ a generalized complex structure on $E, C$ a submanifold and $L$ a maximal isotropic subbundle of $\left.E\right|_{C}$ with $\pi(L)=T C$. Suppose that $\mathcal{J}\left(N^{*} C\right) \cap \pi^{-1}(T C)$ is contained in $L$ and has constant rank. Then there exists a submanifold $\tilde{M}$ (containing $C$ ) which inherits a generalized complex structure $\tilde{\mathcal{J}}$ from $M$, and so that $\tilde{L}$ satisfies $\tilde{\mathcal{J}}\left(\tilde{N}^{*} C\right) \subset \tilde{L}$. Here $\tilde{L}$ is the pullback of $L$ to $\tilde{M}$ and $\tilde{N}^{*} C$ the conormal bundle of $C$ in $\tilde{M}$.

Further assume that $\left[\Gamma\left(L \cap \pi^{-1}(\mathcal{F})\right), \Gamma(L)\right] \subset \Gamma(L)$, where $\mathcal{F}:=\sharp N^{*} C \cap T C$ is the characteristic distribution of $C$. Then $\left[\Gamma\left(\tilde{L} \cap \tilde{\pi}^{-1}(\mathcal{F})\right), \Gamma(\tilde{L})\right] \subset \Gamma(\tilde{L})$. Hence, $(C, \tilde{L})$ is a weak brane in $(\tilde{M}, \tilde{\mathcal{J}})$.

Proof. Since the intersection of $\mathcal{J}\left(N^{*} C\right)$ and $\pi^{-1}(T C)$ has constant rank, the same holds for their sum and for $\pi\left(\mathcal{J}\left(N^{*} C\right)+\pi^{-1}(T C)\right)=\sharp N^{*} C+T C$. Hence, $C$ is a pre-Poisson submanifold $[\mathbf{5}]$ of $(M, \Pi)$. Fix any complement $R$ of $\sharp N^{*} C+T C$ in $\left.T M\right|_{C}$; by Theorem 3.3 of [5], "extending" $C$ in direction of $R$ we obtain a submanifold $\tilde{M}$ of $M$ which is cosymplectic. By Lemma 7.17, we know that $\tilde{M}$ is endowed with a generalized complex structure $\tilde{\mathcal{J}}$. Further, by the same lemma $\mathcal{J} K \cap K^{\perp}$ is trivial. The projection $K^{\perp} \rightarrow$ $K^{\perp} / K$ (for $K=N^{*} \tilde{M}$ ) maps $\mathcal{J} K^{\perp} \cap K^{\perp}$ isomorphically onto $K^{\perp} / K$, and $\tilde{\mathcal{J}}$ is induced by the action of $\mathcal{J}$ on $\mathcal{J} K^{\perp} \cap K^{\perp}$. Therefore, denoting by $\tilde{L}:=L / K$ the pullback of $L$ to $\tilde{M}$, requiring $\tilde{\mathcal{J}}\left(\tilde{N}^{*} C\right) \subset \tilde{L}$ is equivalent to requiring that $\mathcal{J}\left(N^{*} C \cap\left(\mathcal{J} K^{\perp} \cap K^{\perp}\right)\right)$ maps into $\tilde{L}$ under $K^{\perp} \rightarrow K^{\perp} / K$, which in turn means $\mathcal{J}\left(N^{*} C\right) \cap K^{\perp} \subset L$. Now using $K^{\perp}=\pi^{-1}(T \tilde{M})$, $\left.T \tilde{M}\right|_{C}=R \oplus T C$, and recalling that $R$ was chosen so that $R \oplus\left(\sharp N^{*} C+\right.$ $T C)=\left.T M\right|_{C}$, it follows that $\mathcal{J}\left(N^{*} C\right) \cap K^{\perp}=\mathcal{J}\left(N^{*} C\right) \cap \pi^{-1}(T C)$. So our assumption ensures that $\tilde{\mathcal{J}}\left(\tilde{N}^{*} C\right) \subset \tilde{L}$.

Finally notice that the projection $K^{\perp} \rightarrow K^{\perp} / K$ maps $L$ onto $\tilde{L}$. Since $\pi^{-1}(\mathcal{F})$ is mapped onto $\tilde{\pi}^{-1}(\mathcal{F})$, we also have that $L \cap \pi^{-1}(\mathcal{F})$ is mapped onto $\tilde{L} \cap \tilde{\pi}^{-1}(\mathcal{F})$. Hence, our assumption $\left[\Gamma\left(L \cap \pi^{-1}(\mathcal{F})\right), \Gamma(L)\right] \subset \Gamma(L)$ implies $\left[\Gamma\left(\tilde{L} \cap \tilde{\pi}^{-1}(\mathcal{F})\right), \Gamma(\tilde{L})\right] \subset \Gamma(\tilde{L})$.

\section{References}

[1] A. Alekseev and P. Xu, Derived brackets and Courant algebroids, preprint available at: http://www.math.psu.edu/ping/papers.html. 
[2] J. Barton and M. Stienon, Generalized complex submanifolds, arXiv:math.DG/ 0603480 .

[3] H. Bursztyn, G.R. Cavalcanti and M. Gualtieri, Reduction of Courant algebroids and generalized complex structures. Adv. Math. 211(2) (2007), 726-765.

[4] I. Calvo, F. Falceto and M. Zambon, Reduction of Dirac structures along isotropic subbundles, arXiv:math.DG/0702025.

[5] A. Cattaneo and M. Zambon, Coisotropic embeddings in Poisson manifolds, to appear in Trans. AMS.

[6] T.J. Courant, Dirac manifolds, Trans. Amer. Math. Soc. 319(2) (1990), 631-661.

[7] M. Gualtieri, Generalized complex geometry, arXiv:math.DG/0401221.

[8] - Generalized complex geometry, arXiv:math/0703298.

[9] N. Hitchin, Instantons, Poisson structures and generalized Kaehler geometry, Commun. Math. Phys. 265 (2006), 131-164 arXiv:math.DG/0503432.

[10] S. Hu, Hamiltonian symmetries and reduction in generalized geometry, arXiv:math.DG/0509060.

[11] - Reduction and duality in generalized geometry, arXiv:math.DG/0512634.

[12] S. Hu and B. Uribe, Extended manifolds and extended equivariant cohomology, arXiv:math.DG/0608319.

[13] A. Kapustin and D. Orlov, Remarks on A-branes, mirror symmetry, and the Fukaya category, J. Geom. Phys. 48(1) (2003), 84-99.

[14] Y. Lin and S. Tolman, Reduction of twisted generalized Kähler structure, arXiv:math.DG/0510010.

[15] — Symmetries in generalized Kähler geometry, Comm. Math. Phys. 268(1) (2006), 199-222.

[16] J. Marsden and A. Weinstein, Reduction of symplectic manifolds with symmetry. Rep. Math. Phys. 5(1) (1974), 121-130.

[17] P. Ševera and A. Weinstein, Poisson geometry with a 3-form background, Progr. Theoret. Phys. Suppl. 144 (2001), 145-154. Noncommutative geometry and string theory (Yokohama, 2001).

[18] M. Stienon and P. Xu, Reduction of generalized complex structures, arXiv:math.DG/0509393.

[19] I. Vaisman, Isotropic subbundles of $T M \oplus T^{*} M$, Int. J. Geom. Methods Mod. Phys. 4(3) (2007), 487-516.

[20] - Reduction and submanifolds of generalized complex manifolds, Differ. Geom. Appl. 25(2) (2007), 147-166.

[21] J. Winkelmann, Surface foliations with compact complex leaves are holomorphic, arXiv:math.CV/0409049.

[22] P. Xu, Dirac submanifolds and Poisson involutions, Ann. Sci. École Norm. Sup. (4) 36(3) (2003), 403-430.

INSTITUT FÜR MATHEMATIK

UNIVERSITÄT ZÜRICH-IRCHEL

WINTERTHURERSTR. 190

CH-8057 ZüRICH, SwitZERLAND

E-mail address: marco.zambon@math.unizh.ch 
Received 02/06/2007, accepted 07/03/2008

I am very indebted to Henrique Bursztyn for clarifying to me some of the constructions of [3] and to Marco Gualtieri for some crucial discussions at the Geometry Conference in Honor of Nigel Hitchin (September 2006), during which he clarified conceptual issues and also suggested some of the techniques used in this paper. Furthermore, I thank A. Cattaneo, G.R. Cavalcanti, F. Falceto, B. Scardua, and M. Stienon for discussions. I also thank A. Cattaneo for supporting my attendance to conferences relevant to this work and F. Falceto for a visit to the Universidad de Zaragoza where part of this work was done. Further, I acknowledge support from the Forschungskredit of the Universität Zürich and partial support of SNF Grant no. 20-113439. This work has been partially supported by the European Union through the FP6 Marie Curie RTN ENIGMA (Contract number MRTN-CT-2004-5652) and by the European Science Foundation through the MISGAM program. 